\title{
Investigation of Aberrantly Methylated Differentially Expressed Genes in Pancreatic Cancer by Integrated Bioinformatics Analysis
}

\section{Cailin xue}

Nanjing Medical University

\section{Peng gao}

The Affiliated Changzhou No. 2 People's Hospital of Nanjing Medical University

\section{Xudong zhang}

The Affiliated Changzhou No. 2 People's Hospital of Nanjing Medical University

\section{Xiaohan cui}

Nanjing Medical University

Lei jin

The Affiliated Changzhou No. 2 People's Hospital of Nanjing Medical University

\section{Chunfu zhu}

The Affiliated Changzhou No. 2 People's Hospital of Nanjing Medical University

Xihu qin ( $\square$ zcfmlm@njmu.edu.cn )

The Affiliated Changzhou No. 2 People's Hospital of Nanjing Medical University

\section{Research Article}

Keywords: pancreatic cancer, microarray analysis, gene expression, methylation, biomarker

Posted Date: January 4th, 2021

DOl: https://doi.org/10.21203/rs.3.rs-132250/v1

License: (c) (i) This work is licensed under a Creative Commons Attribution 4.0 International License. Read Full License 


\title{
Investigation of aberrantly methylated differentially expressed genes in pancreatic cancer by integrated bioinformatics analysis
}

\author{
Cailin Xue ${ }^{1,2}$, Peng Gao ${ }^{1}$, Xudong Zhang ${ }^{1,2}$, Xiaohan Cui ${ }^{1,2}$, Lei Jin ${ }^{1}$, Chunfu Zhu ${ }^{1 凶}$, Xihu \\ Qin ${ }^{1}{ }^{凶}$
}

1. Department of Hepatobiliary Surgery, The Affiliated Changzhou No. 2 People's Hospital of Nanjing Medical University, Changzhou, Jiangsu 213003, P.R. China.

2. Nanjing Medical University, Nanjing, Jiangsu, 211166, P.R. China

${ }^{\square}$ Corresponding author:

Zhu chunfu, Department of Hepatobiliary Surgery, The Affiliated Changzhou No. 2 People's Hospital of Nanjing Medical University, Changzhou, Jiangsu 213003, P.R. China E-mail: qxhnjmu@163.com

Qin xihu, Department of Hepatobiliary Surgery, The Affiliated Changzhou No. 2 People's Hospital of Nanjing Medical University, Changzhou, Jiangsu 213003, P.R. China E-mail: zcfmlm@njmu.edu.cn.

Background: Abnormal methylation of DNA sequences plays an important role in the development and progression of pancreatic cancer (PC). The purpose of this study was to identify abnormal methylation and expression genes and related signaling pathways in PC by comprehensive bioinformatic analysis of three datasets in the Gene Expression Omnibus (GEO).

Methods: Datasets of gene expression microarrays (GSE91035, GSE15471) and gene methylation microarrays (GSE37480) were downloaded from the GEO database. Differentially-methylated-differentially expressed genes (MeDEGs) were analysis by GEO2R software. GO and KEGG enrichment analyses of selected genes were performed using DAVID database. A protein-protein interaction (PPI) network was constructed by STRING and visualized in Cytoscape. Core module analysis was performed by Mcode in Cytoscape. Hub genes were obtained by CytoHubba app. in Cytoscape software.

Results: A total of 267 hypomethylation-high expression genes, which were enriched in biological processes of cell adhesion, biological adhesion and regulation of signaling were obtained. KEGG pathway enrichment showed ECM-receptor interaction, Focal adhesion and PI3K-Akt signaling pathway. The top 5 hub genes of PPI network were EZH2, CCNA2, CDC20, KIF11, UBE2C. As for hypermethylation-low expression genes, 202 genes were identified, which were enriched in biological processes of cellular amino acid biosynthesis process and positive regulation of PI3K activity, etc. The pathways enriched were the pancreatic secretion and biosynthesis of amino acids pathways, etc. The five significant hub genes were DLG3, GPT2, PLCB1, CXCL12 and GNG7. In addition, five genes, including 
CCNA2, KIF11, UBE2C, PLCB1 and GNG7, significantly associated with patient's prognosis were also identified.

Conclusion: Novel genes with abnormal expression were identified, which will help us further understand the molecular mechanism and related signaling pathways of $\mathrm{PC}$, and these aberrant genes could possibly serve as biomarkers for precise diagnosis and treatment of PC.

Keywords: pancreatic cancer, microarray analysis, gene expression, methylation, biomarker

\section{Introduction}

Pancreatic carcinoma (PC) is one of most common malignant tumors with high incidence and poor prognosis. According to the American Cancer Society, there're approximately 55,440 newly diagnosed cases and 44,330 PC-related deaths in the US in 2018[1]. Although chemotherapy and surgical method can be implemented, the average five-year survival rate of PC is only $8 \%$ which has been improved poorly over the decade years ranking the lowest among all the malignant tumors[1]. Multiple abnormally expressed genes and signaling pathways have been reported to play critical roles in the development of PC[2, 3]. In additional, epigenetics changes have been verified to affect its progression[4].

Tumor epigenetics is an inherited modification which affects the gene expression without alternating tsDNA sequence[5]. Tumor epigenetics mainly includes the methylation of DNA and modifications of histone tail[6]. DNA methylation, as one of the vital epigenetic changes, is highly associated with the development of numerous digestive cancers especially for PC[7, 8]. Aberrant methylation of the gene promoter plays a critical role in the carcinogenesis of the cancer by affecting gene expression[3,9]. At the same time, DNA methylation is also a molecular marker for the diagnosis of pancreatic cancer[10], which can help greatly in the early diagnosis of the cancer. Therefore, identification of abnormal methylation gene and discovering the function of the gene are of great significance in providing a better understanding of the role of methylation in the progression of PC.

Nowadays, with the usage of microarrays based on the high-throughput sequencing platforms, identifying aberrant genes and understanding the pathogenesis of tumor become more effective and convenient. In the past few years, lots of differentially expressed genes (DEGs) and aberrant methylation genes (DMGs) have been discovered in various microarray profiles. However, the relationship between different gene and aberrant methylation gene remains unclarified.

In this study, we obtained the gene database profiles (GSE91035, GSE62165) and aberrant methylation profiles (GSE37480) from the Gene Expression Omnibus (GEO) database. The abnormal expression and methylation gene were discovered through integrating and analyzing the database profiles by the bioinformatic online tools and software. Connection between the aberrant genes and hub genes were revealed through the construction of the PPI network. The aim of the study is to find the novel gene with alternation methylation, and to investigate their mechanism in the pathogenesis of pancreatic cancer (PC).

\section{Materials and method}

Microarray data 
In this study, the gene expressions profiling dataset (GSE91035, GSE62165) and gene methylation profiling dataset (GSE37480) were downloaded from the GEO database (https://www.ncbi.nlm.nih.gov/geo/). The dataset GSE91035 which was produced on the platform GPL22763 (Agilent-039714 LincRNA SurePrint G3 Human GE 8 x 60K Microarray PVD 028004) includes 27 human PDAC and 8 normal pancreatic tissues. The dataset GSE62165 containing 118 PDAC tissues and 13 normal pancreatic tissues was produced on the platforms GPL13667 (Affymetrix Human Genome U219 Array). The methylation gene dataset GSE37480 includes 7 PDAC and 4 normal tissues, and the methylation genes dataset was produced on the platform GPL9767 (Agilent-014791 Human CpG Island ChIP-on-Chip Microarray 244K).

\section{Data processing}

The GEO2R online software was used to analyze the raw submitter supplied data of microarrays (GSE91035, GSE62165 and GSE37480) and identify differentially expressed genes (DEGs) and differentially methylated genes (DMGs). GEO2R is a widely used web tool which can be used to compare different groups of samples in a GEO series, with the purpose of screening DEGs genes under the experimental conditions. $\mathrm{P}<0.05$ and $|\log \mathrm{FC}|>1$ were applied as the cut-off criteria to find DEGs. And the cut-off criteria in finding DMGs were $\mathrm{P}<$ 0.05 and $|t|>1$. Finally, Venn diagram (http://bioinformatics.psb.ugent.be/webtools/Venn/) was used to identify the hypomethylation and high-regulated genes by overlapping the high DEGs in GSE91035 and GSE62165 and the high DMGs in GSE37480. The hypermethylation and down-regulated genes were retrieved similarly as above.

Functional and pathway enrichment analysis

The Database for Annotation, Visualization and Integrated Discovery (DAVID) and $\mathrm{R}$ package clusterProfiler were applied to perform the Gene Ontology (GO) analysis and Kyoto Encyclopedia of Genes and Genomes (KEGG) pathway enrichment analysis for the genes[11, 12]. The Gene ontology (GO) analysis was composed of the cellular component, molecular function, and biological process. $\mathrm{P}<0.05$ was regarded as statistical significance.

PPI network construction and module analysis

STRING database (Search Tool for the Retrieval of Interacting Genes) was used to construct PPI network of genes, and an interaction with a combined score $>0.4$ was regarded as statistically significant. The Visualization of the PPI network was realized by the Cytoscape software. Then MOCDE was used in Cytoscape to screen the PPI network with the MCODE scores $>5$, degree cut-off $=2$, node score cut-off $=0.2$, Max depth $=100$ and $\mathrm{k}$-score $=2$. Hub genes were selected with the connection degree $>10$ in the HUBITT integrated in Cytoscape. The functional enrichment analysis of the genes in module was achieved in $\mathrm{R}$ package clusterProfiler with a significant threshold of $\mathrm{P}<0.05$.

\section{Validation of hub genes}

Oncomine is online microarray analysis database based on RNA sequencing of the tumor. In this study, the Oncomine were used to investigate the hub gene expression between the tumor and normal tissues. The TCGA database comprises comprehensive, multidimensional maps of 
key cancer genome alterations among various cancers. MEXPRESS (http://mexpr ess.be) is a database which was used for analysis the DNA methylation based on the TCGA database. In this study, the MEXPRESS was used to validate hypomethylation-high expression genes and hypermethylation-low expression genes.

Genetic alterations and survival analysis of hub genes

The cBioPortal (http://www.cbioportal.org/) is a visual web which can provide visualization, online analysis and downloading genome data of various cancer based on the TCGA database. Besides, the cBioPortal tool can be used to analysis the relationship between gene expression and the methylation status. The Cancer Genome Atlas (TCGA) is a collaboration between the National Cancer Institute (NCI) and National Human Genome Research Institute (NHGRI), which has generated comprehensive, multi-dimensional maps of the key genomic changes in 33 types of cancer. GEPIA is web server for analyzing the RNA sequencing expression data of 9,736 tumors and 8,587 normal samples from the TCGA by using a standard processing pipeline[13]. To analyze the effect of hub gene on the prognosis of patients with pancreatic cancer, the GEPIA database was used to analyze the prognosis of patients in the TCGA database.

\section{Result}

Identification of aberrantly methylated-differentially expressed genes in PDAC

The GEO2R was used to analyze the DEGs or DMGs in the microarrays (GSE91035, GSE62165 and GSE37408). A total of 5031 high-expression genes were found in the datasets (2300 in GSE91035 and 2731 in the GSE62165), and 2989 low-expression genes were obtained from the datasets (1598 in the GSE91035 and 1391 in the GSE62165). In the methylation dataset GSE37408, 4216 hypomethylation genes and 5335 hypermethylation genes were identified, respectively. 267 high-expression-hypomethylation genes were found by overlapping the high-expression and hypomethylation genes, while 202 low-expression-hypermethylation genes were obtained by overlapping the low-expression and hypermethylation genes (Fig. 1). The expression of the top 25 high-expression-methylation genes and top 25 low-expression-hypomethylation genes in the GSE37408 were shown in the Fig. 2.

GO and pathway functional enrichment analysis

GO and KEGG enrichment analyses were performed by R package clusterProfiler and DAVID software was used to explore the function of the different expression genes. As showed in Table 1, the high-expression-hypomethylation genes were enriched in biological processes (BP) of cell adhesion, biological adhesion and regulation of signaling. Molecular function (MF) showed that these genes were correlated with kinase activity, cell adhesion molecule binding and protein complex binding. As for cell component (CC), the enrichments were mainly in cell-substrate junction and adherens junction. Besides, the KEGG pathway assay showed that the enrichment was mostly involved in focal adhesion (Table 2). 
However, for the low-expression-hypermethylation genes, just as list in Table. 1, the main genes that were enriched were about the $\mathrm{CC}$ of intrinsic component of plasma membrane and bicellular tight junction, and the BP of cellular amino acid biosynthetic process and positive regulation of PI3K activity. The enrichment of MF was mostly in the amino acid transmembrane transporter activity and calmodulin binding. Additionally, KEGG analysis displayed that the genes were associated with the pathway of pancreatic secretion and amino acids biosynthesis (Table 2).

PPI network construction, module analysis and hub gene selection

PPI networks were constructed on the basis of STRING database. Module analysis was conducted by MCODE in Cytoscape software. The hub genes were obtained by using the cytoHubba embedded in the Cytoscape. The PPI network of hypomethylation-high expression genes was showed in the Fig. 3A and the hub genes that were detected included EZH2, CDC20, CCNA2, KIF11 and UBE2C. The top modules were displayed in the Fig.3B. The pathway of the core modules demonstrated the important functions of the cell cycle, ECM-receptor interaction, focal adhesion, and PI3K-Akt signaling pathway (Table 3). As for hypermethylation-high-expression genes, the PPI network was showed in Fig. 4A and the top 5 hub genes were DLG3, GPT2, PLCB1, CXCL12 and GNG7. The core modules of PPI network were displayed in Fig. 4B, C and D, respectively. The pathway associated with the core module was the Chemokine signaling pathway (Table 3).

Validated the hub genes in the TCGA

To further investigate the results, we exam the expression of the hub genes in the Oncomine database. Through analysis the expression of the hub genes, we found the expression of all the hub gene were in consistent with the expression in the GEO datasets (Figure 5 ). For validating the methylation status of the hub genes, the online methylation web MEXPRESS were used to investigate the methylation status, and the results shows the overexpression hub genes have significant hypermethylation status, and the downregulation genes in the GEO datasets have hypomethylation status. All the online validated results were in consistent with our bioinformatics analysis (Figure 6).

Furthermore, in order to verify the expression in protein level of the hub genes, we investigate the immunohistochemical analysis of the hub genes in the Human Protein Atlas database. For the hypomethylated upregulated genes, EZH2, CDC20, CCNA2, KIF11 and UBE2C have a high expression trend in the pancreatic cancer. when it come to the hypermethylated downregulated genes, the results show that these genes were low-expression in the tumor tissue compared with the normal tissue (Figure 7). However, due to the limitation of the number of samples in the Human Protein Atlas database, the experimental results need to be further verified in a large number of clinical samples.

Genetic alterations and survival analysis of hub genes

In addition, we analysis the gene genetic alterations of the hub genes with the usage of cBioPortal tools. The results show that more $30 \%$ of the tumor patient in the cBioPortal have at least one hub gene alteration and the hub gene GNG7 is the most frequently altered gene with a $4 \%$ gene alteration rate (Figure 8 ). Then, we analysis the correlation between the 
mRNA expression of hub genes and the clinic outcome of the patient basing on the TCGA datasets in the GEPIA. The results show that the hypomethylated upregulated genes CDC20, KIF11, UBE2C have a significant negative correlation with the overall survival of the patients. And hypermethylated downregulated genes PLCB1, GNG7 have a significant positive correlation with the overall survival according to the GEPIA databases (Figure 9). However, the hub genes EZH2, CCNA2, DLG3, GPT2, CXCL12 seem to have no significant correlation the outcome of the patient, which need to explore in a large clinic sample.

\section{DISCUSSION}

Pancreatic cancer is a common malignancy of the digestive system with a high mortality rate. The occurrence of pancreatic cancer is a complex biological process, which involves abnormal expression of many tumor-related genes and abnormal activation of signaling pathways[14]. Therefore, the discovery of abnormal expression of genes and related signaling pathways is of great significance for the exploration of the molecular mechanism of tumor genesis. Currently, the use of gene chips and tumor bioinformatics analysis makes it easier to identify abnormal genes and signaling pathways involved in tumor development.

DNA methylation is one of the most important gene epigenetic modifications and is widely involved in tumor development, which was usually occurred on the cytosines that precede a guanine nucleotide[15]. And CPG island usually appears in the promoter region of genes and plays a decisive role in gene expression. Methylation on $\mathrm{CpG}$ can alter the spatial structure of DNA, thus affecting the binding of DNA binding proteins to DNA and suppressing the gene expression[16]. So far, a large number of studies have shown that demethylation of oncogenes and hypermethylation of tumor suppressor genes play important roles in the development of tumors. For example, in breast cancer, the DNA methylation of the CLDN6 promoter can down-regulate the CLDN6 expression and induced the enhancement of the invasion ability of the breast cancer[17]. And in pancreatic cancer, the histone demethylase KDM3A can promotes tumorigenesis of cancer by regulating the expression of DCLK1[18].But previous studies have focused on the effect of a particular methylated gene or an individual's gene methylation profile on tumors. Therefore, systematic analysis of abnormal gene expression and abnormal methylation state can more comprehensively and accurately reveal the effect of methylation on tumor.

As we know, the methylation of gene including suppressor gene and cancer-promoting gene plays an important role in the development of the cancer[7, 19]. The methylation of $\mathrm{CpG}$ island can suppress the expression of gene[20]. In pancreatic cancer, the high-methylation of genes can promote the proliferation and invasion of the cancer[21]. In addition, the malignancy of cancer can be enhanced through the way of methylation.[22] Thus, it can be concluded that the gene methylation has a significant function in the oncogenesis. In recent years, high-throughput GeneChip analysis provides a convenient and effective way to obtain thousands of aberrant gene[23]. In this study, we analyzed the relationship between the different expression -methylation gene and the prognosis of the patients with PADC.

In present study, we identified 267 hypomethylated, upregulated genes and 202 hypermethylated, downregulated gene with the using the bioinformatic tools. Through the GO analysis, we found that the BP of the hypomethylated, upregulated genes were enriched in the cell adhesion, biological adhesion, regulation of signal transduction, cell morphogenesis 
involved in differentiation and regulation of signaling. The $\mathrm{CC}$ analysis showed the enrichments in the focal adhesion, adherens junction, cell-substrate adherens junction. The MF enrichment of the genes were in the kinase activity and cell adhesion molecule binding. It is reasonable that in the early stage of tumorigenesis, the weaken of the attachment of a tumor cell, either to another cell or to an underlying substrate promotes its migration[24]. On morphological changes, the epithelial-mesenchymal transformation (EMT) can promote invasion and migration of the pancreatic cancer cells[25]. In addition, the changes in cellular signaling pathways such as activation of EGFR signal in pancreatic cancer cells are very common during tumor development[26]. The activation of MAPK signaling pathway induced by increased activity of p38 kinase promotes tumor cell proliferation[27]. KEGG pathway enrichment analysis suggested significant enrichments in the pathways of ECM-receptor interaction, PI3K-Akt signaling pathway and focal adhesion. These results are consistent with the facts that the up-regulation of PI3K-Akt signaling pathway promotes the pancreatic cancer[28].

The overview function connection of the hypomethylated, upregulated genes gene was illustrated by PPI network. The top 5 hub genes obtained from the PPI network included EZH2, CDC20, CCNA2, KIF11 and UBE2C. The high expression of EZH2, a histone methyltransferase, promotes EMT transformation of pancreatic cancer and also the tumor metastasis, which is negatively correlated with the prognosis of patients[29, 30]. This result is consistent with what were found in the gene enrichment analysis. The CDC20 is an anaphase-promoting complex activator, the high expression of CDC2 is associated with pancreatic cancer differentiation and progression[31]. The CCNA2, a regulator of the cell cycle, is usually abnormally high expressed in cancer cell and can enhance the cell proliferation[32]. The high-regulation of KIF11 in glioblastoma can enhance the invasion and proliferation[33]. In oral cancer and lung cancer, the KIF11 can treated as a prognostic indicator[34, 35]. UBE2C is an oncogene in breast cancer and gastric cancer, and the increased expression is negatively correlated with the prognosis of patients[36, 37]. However, the relationships between the expression of KIF11 and UBE2C with pancreatic cancer is still unclear and need further study.

For the hypermethylated, downregulated genes, BP analysis showed the genes were mainly enriched in the progresses of nervous system development, and cellular amino acid biosynthetic process. Neural infiltration is a major factor in causing the poor prognosis of pancreatic cancer. Abnormal expression of neural development-related genes promotes neural invasion of pancreatic cancer, while the neural ablation can enhance the prognosis[38]. Abnormal amino acid metabolism is common in the pancreatic cancer cells. The inhibition of glutamine metabolism can suppress Pancreatic Cancer[39]. The CC analysis of the genes showed the enrichments in the neuron part, bicellular tight junction and the intrinsic component of plasma membrane. The KEGG analysis showed the enrichments were in the amino acid transmembrane transporter activity, fibroblast growth factor-activated receptor activity. The results are reasonable that the weaken of the bicellular junction can promote the immigration of the cells, and it had been found that the down-regulation of tight junction-associated MARVEL protein marvelD3 can promote the transformation of EMT[40] and activity decline of the amino acid transmembrane transporter such as folic acid transporter can result in a disorder in the metabolism in cancer. 
PPI network of hypermethylated, downregulated genes illustrated the overview of their functional connections, of which top 5 hub genes were also selected: DLG3, GPT2, PLCB1, CXCL12 and GNG7. DLG3, a tumor suppressor, encodes a member of the membrane-associated guanylate kinase protein family. And the down-regulation of DLG3 promotes the invasion and proliferation in glioblastoma, however, in pancreatic cancer, the function of DLG3 has not been reported[41]. CXCL12 plays a role as a tumor suppressor gene in pancreatic cancer, the up-regulation of the CXCL12 suppresses human pancreatic cancer growth and metastasis[42]. The loss of PLCB1 can induce cell damage under oxidative stress and enhance the alpha-synuclein aggregation[43], but the function in the process of pancreatic cancer formation still needs further study. In breast cancer, the expression level of GPT2 is significantly increased, and is related to the pathological grade[44].

Module analysis for the hypermethylated, downregulated gene indicates that the cell cycle, ECM-receptor interaction, focal adhesion, and PI3K-Akt signaling pathway may play great roles in the development of pancreatic carcinoma. It is well-known that the cell cycle is the critical cellular processes with DNA replication and translation and it's arrest can inhibit cell proliferation[45]. As we all know, the adhesion function of cells is the primary step for tumor metastasis[46], and the adhesion function enhanced by the focal adhesion and ECM-receptor interaction can accelerate cancer cell metastasis in the blood vessels. The PI3K signaling pathway is activated in a variety of tumors including pancreatic cancer, and the activation of PI3K signaling pathway promotes the metastasis of tumor cells[47].

\section{Conclusion}

In summary, through bioinformatic analyses of gene expression and methylated profile in the microarray datasets, we have discovered a series of genes that are abnormally methylated and expressed in the cancer cell of PADC patients. Five survival-related hub genes (DLG3, GPT2, PLCB1, CXCL12 and GNG7) were found. These results help us to understand the role of apparent modification in the development of pancreatic cancer and can also be used as indicators for early diagnosis and prognosis evaluation of pancreatic cancer. However, the data in this paper needs to be further verified in biological experiments.

Ethics approval and consent to participate :Not applicable

Consent for publication : Not applicable

Availability of data and materials : The datasets GSE91035, GSE62165 and GSE37480 are available in the Gene Expression Omnibus (GEO, https ://www.ncbi.nlm.nih.gov/ geo/).

Competing interests: The authors report no conflicts of interest in this work

Funding:This study was supported by grants from the National Natural Science Foundation of China (grant no. 81672469). The Social Development Foundation of Science and Technology of Jiangsu (grant no. BE2016658)

Author Contributions: Xihu Qin and Chunfu Zhu contributions to conception and design. Cailin Xue, Peng Gao, Xudong Zhang, Xiaohan Cui performed the data mining. Cailin Xue wrote the manuscript. All the authors have read and approved the submission of the manuscript.

Acknowledgments: Not applicable. 
FIGURE 1 Identification of aberrantly methylated-differentially expressed genes in gene expression datasets (GSE62165, GSE91035) and gene methylation dataset (GSE37048). a. hypomethylation and up-regulated genes; b. hypermethylation and down-regulated genes

A

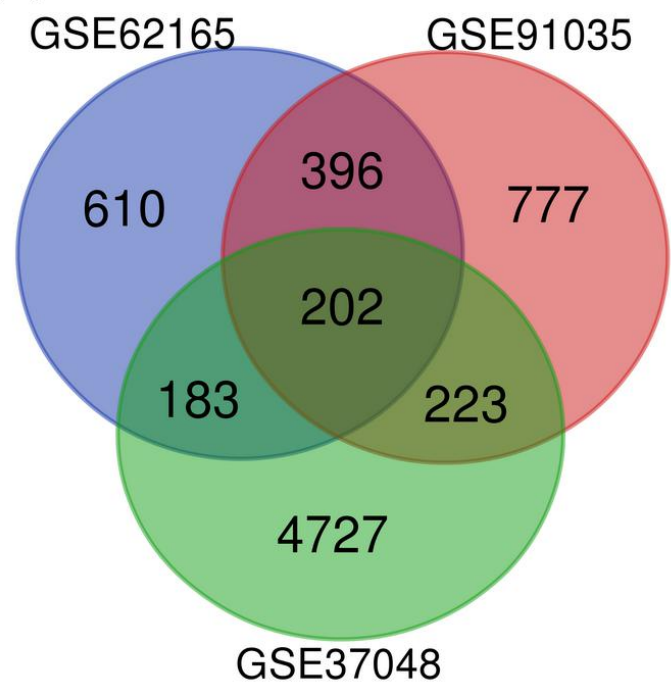

B

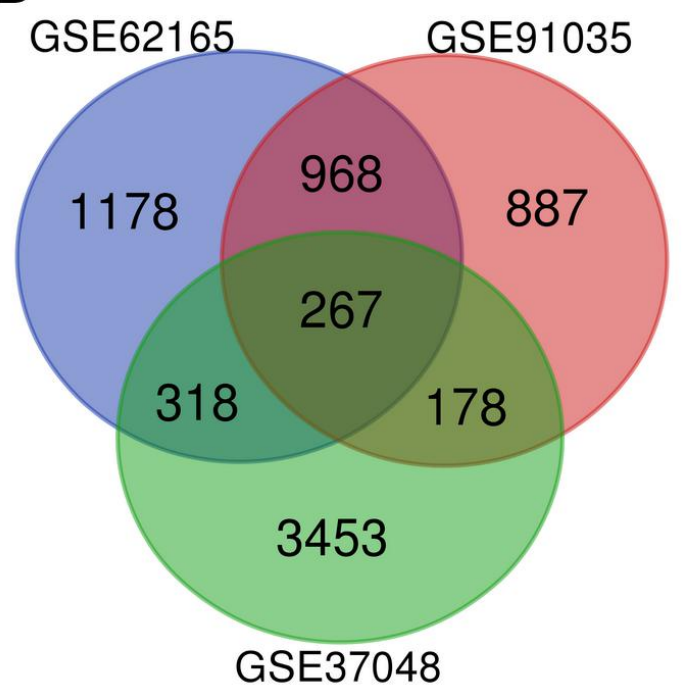


FIGURE 2 Representative heat map of the top 100 differentially expressed genes in dataset GSE37048 (50 up-regulated genes and 50 down-regulated genes). X-axis represents samples, Y-axis represents genes, red stands for upregulation, and green stands for downregulation

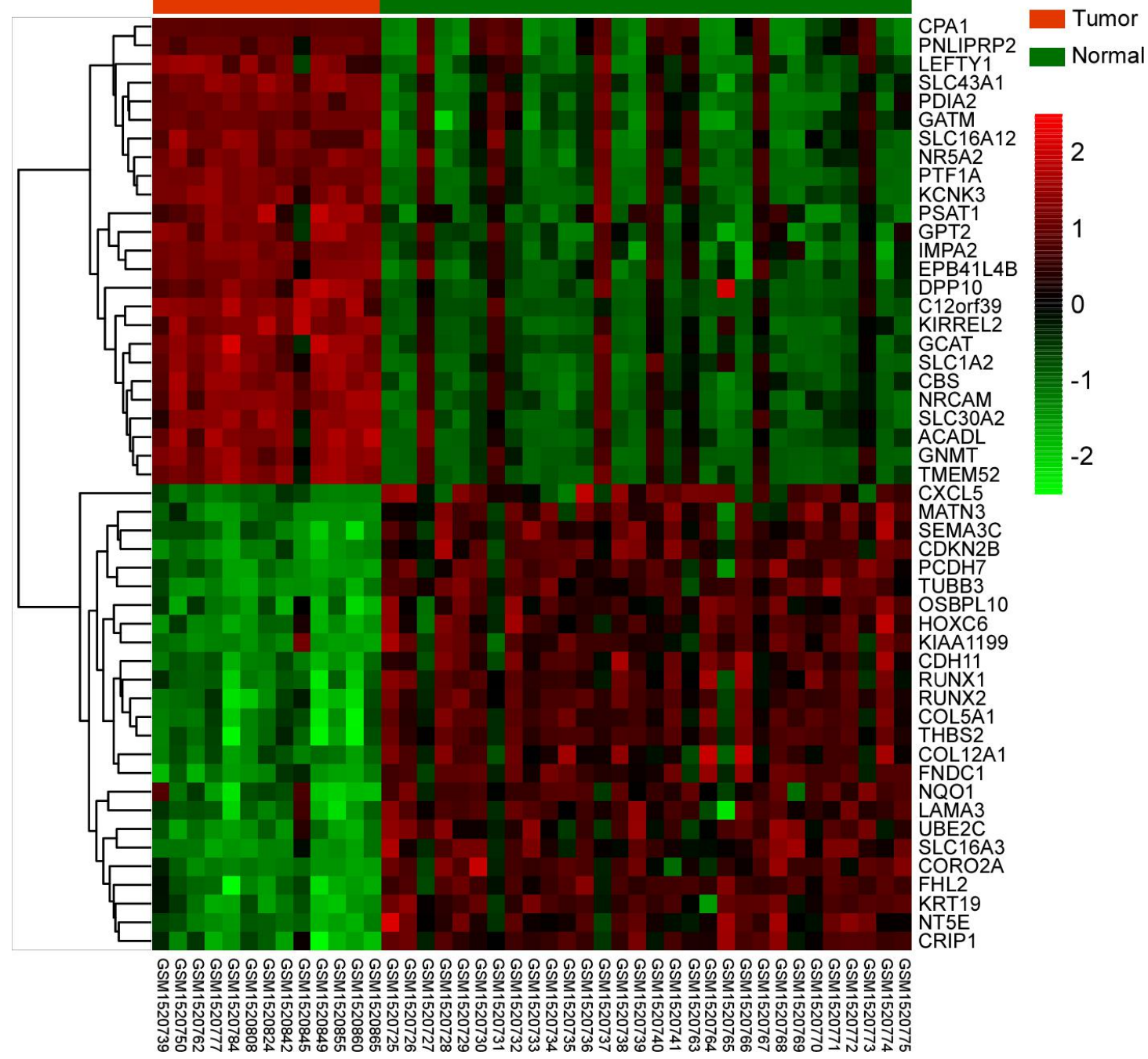


FIGURE 3 PPI network and modules of hypermethylation-low expression genes. a. PPI network; b. top module.

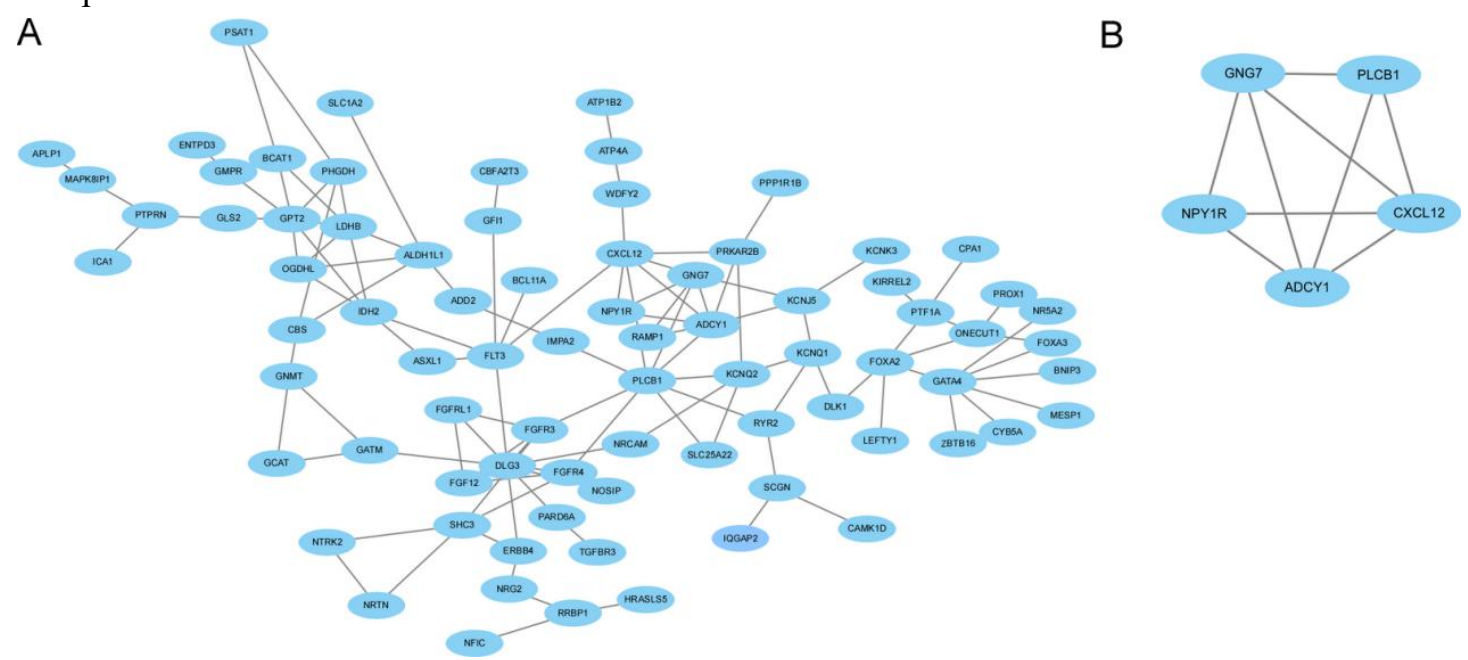

FIGURE 4 PPI network and three modules of hypomethylation-high expression on genes.

a. PPI network; b.c.d. top module
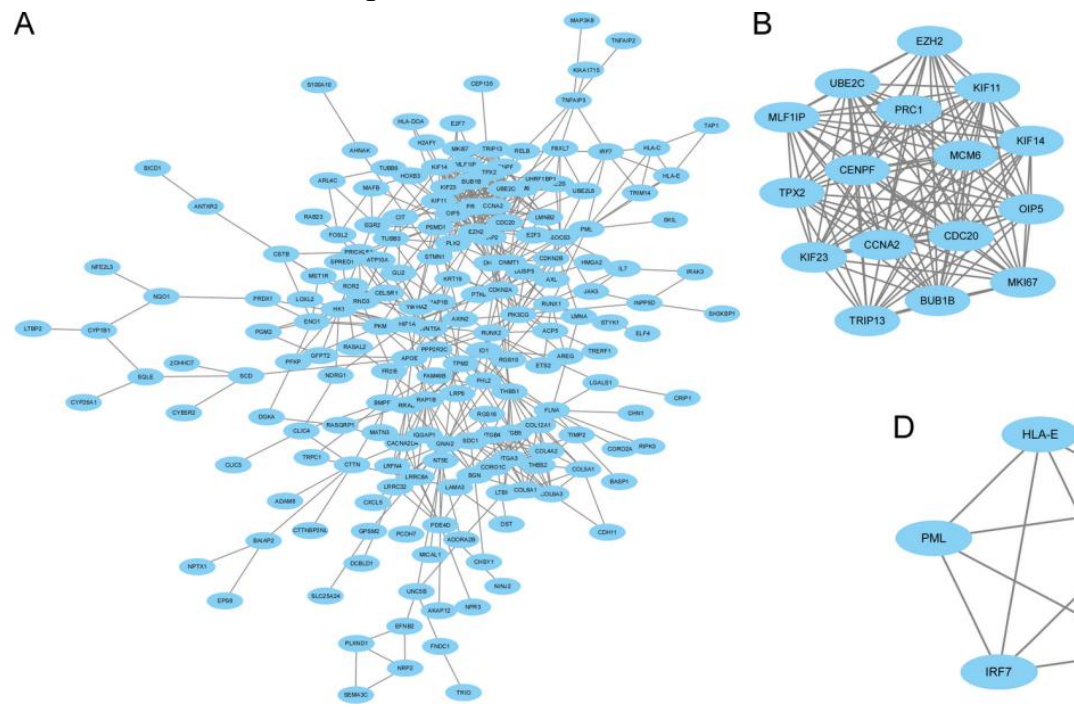

C
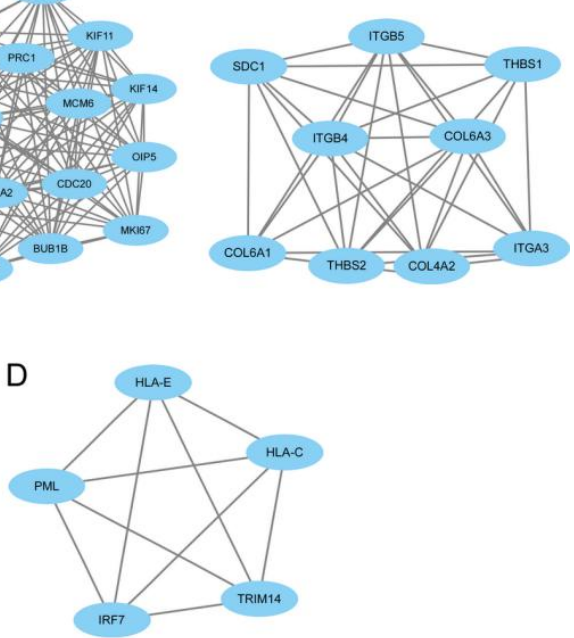
FIGURE 5 Validation of the expression of hub genes in Oncomine database. The mRNA expression levels of (a) EZH2, (b) CDC20, (c) CCNA2, (d) KIF11, (e) UBE2C were upregulated in the tumor compared to the normal tissues. And the mRNA expression levels of (f) DLG3, (g) GPT2, (h) PLCB1, (i) CXCL12, (j) GNG7 were down-regulated in the tumor tissues compared to the normal tissues according to the Oncomine databases.
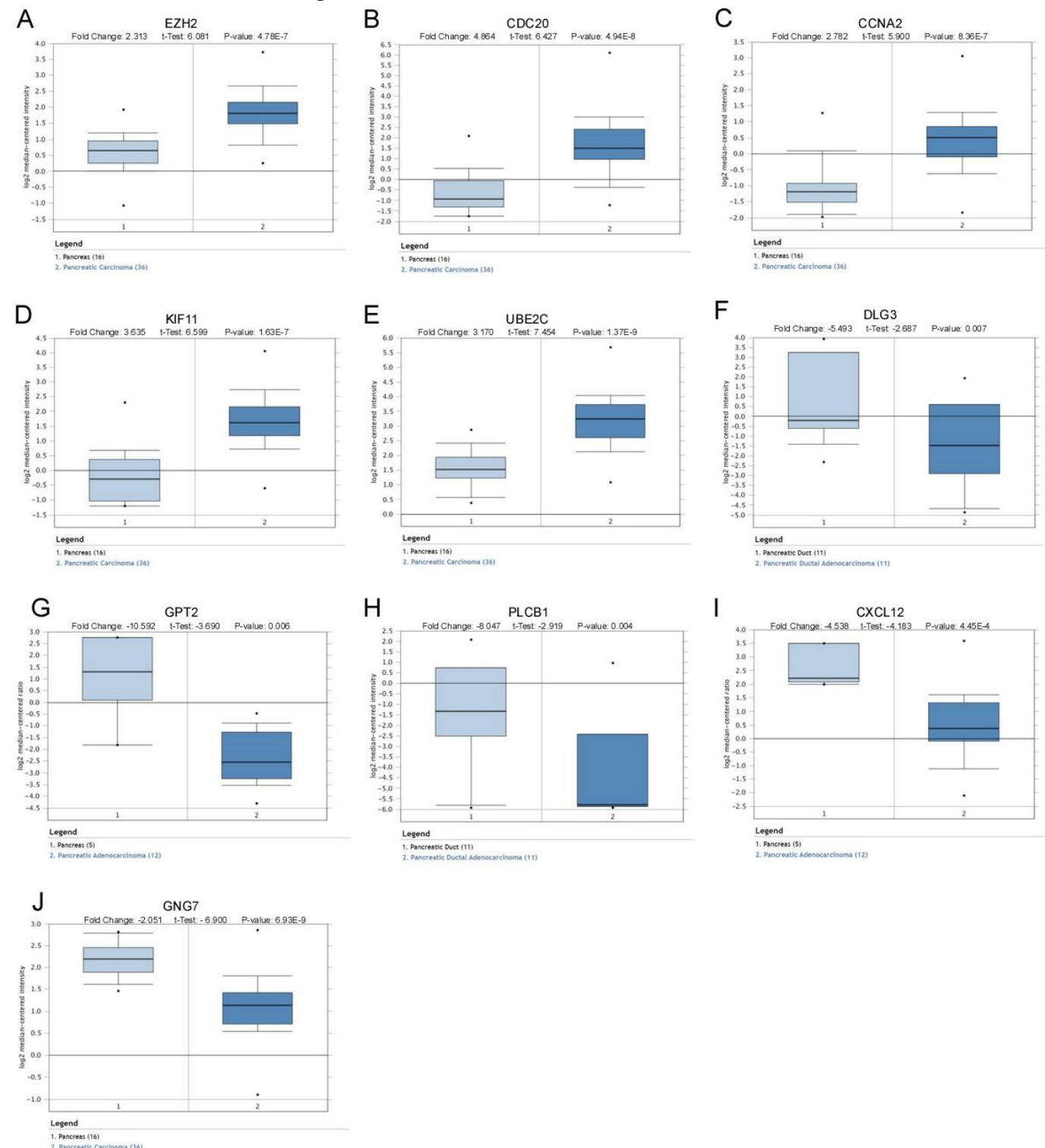
FIGURE 6 Verification of the methylation levels of 5 hypomethylated upregulated genes and 5 hypermethylated downregulated genes in MEXPRESS databases. a EZH2. b CDC20. c CCNA2. d KIF11. e UBE2C. f DLG3. g GPT2. h PLCB1. i CXCL12. j GNG7

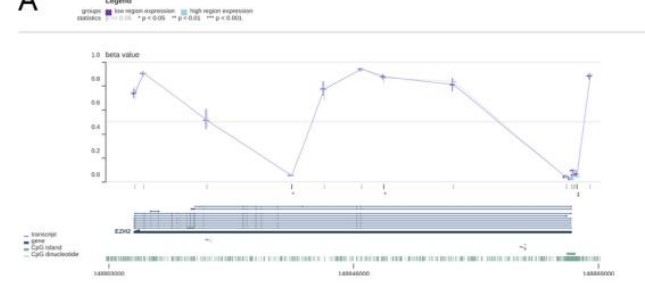

B

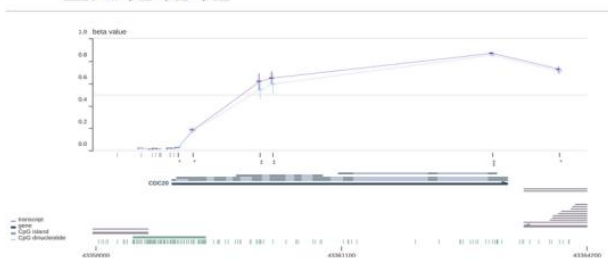

$\mathrm{C}=\mathrm{s}$

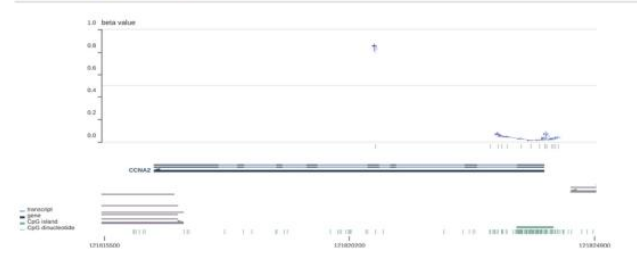

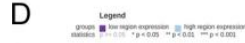

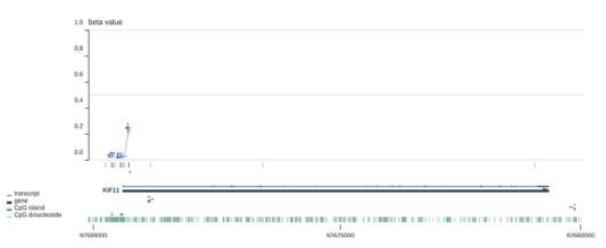

E 다에

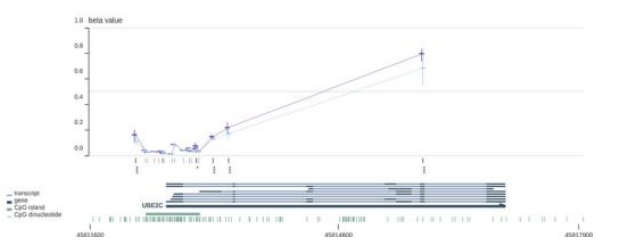

$\mathrm{F}$

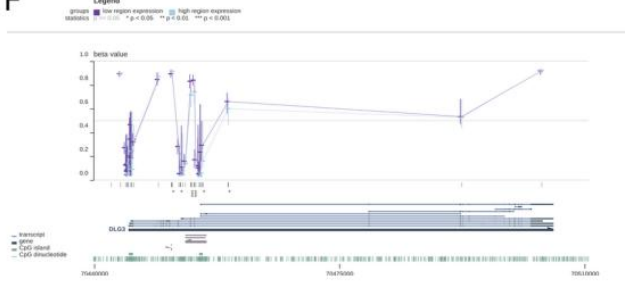

G

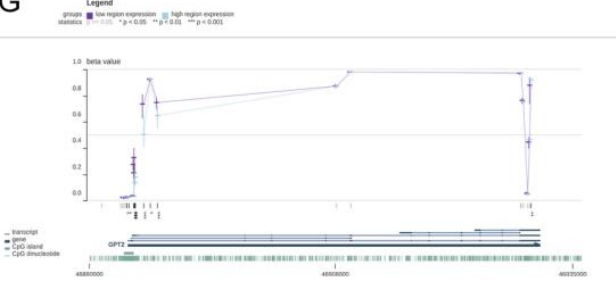

$\mathrm{H}=$

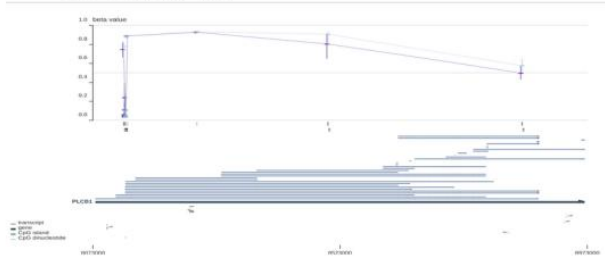

$1 \quad=$

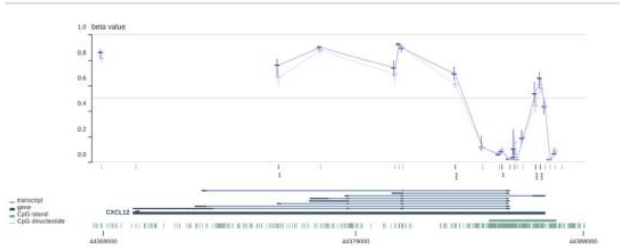

$\mathrm{J}=$

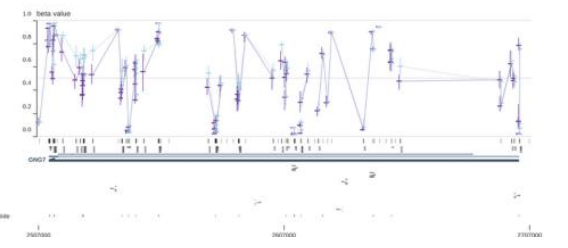


FIGURE 7 Validation of hub genes on protein level in the Human Protein Atlas database.

The protein levels of (a) EZH2, (b) CDC20, (c) CCNA2, (d) KIF11, (e) UBE2C were upregulated in the tumor compared to the normal tissues. And the protein levels of (f) DLG3, (g) GPT2, (h) PLCB1, (i) CXCL12, (j) GNG7 were down-regulated in the tumor tissues compared to the normal tissues according to the database

A

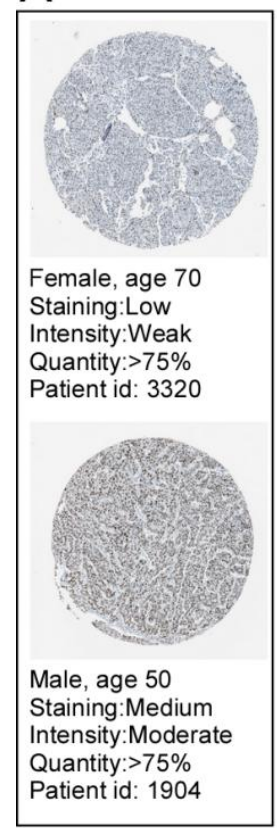

$\mathrm{F}$

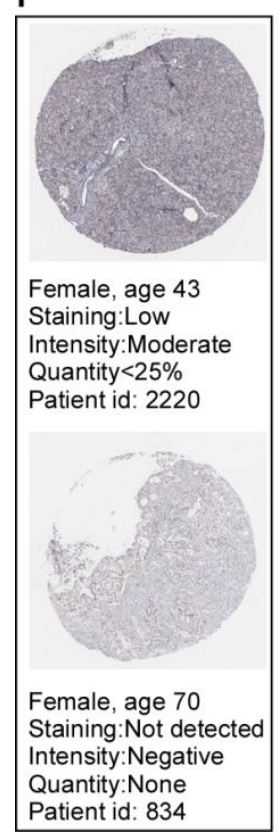

B

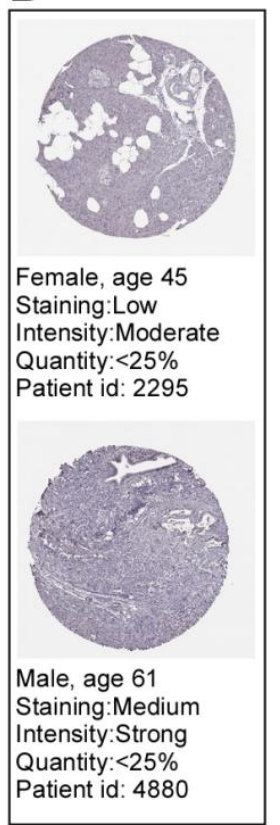

G

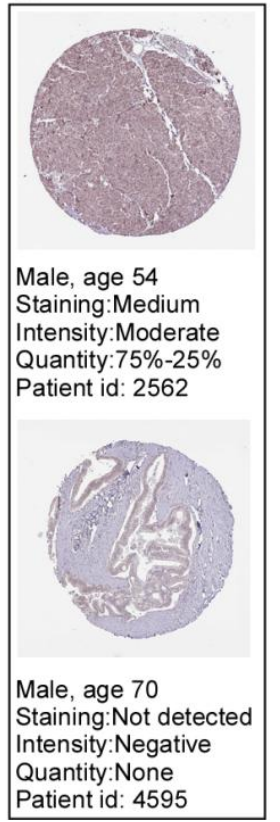

C

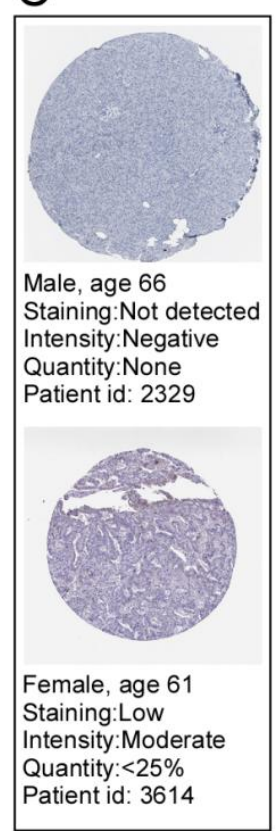

$\mathrm{H}$

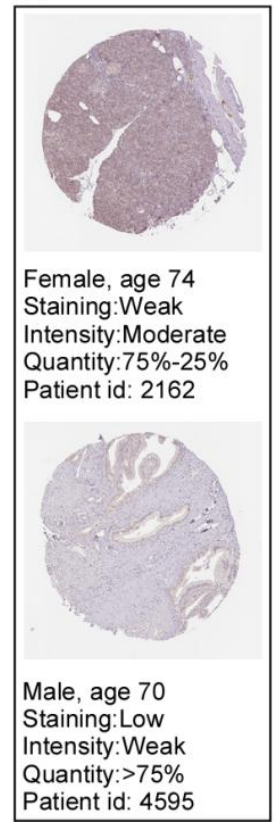

D

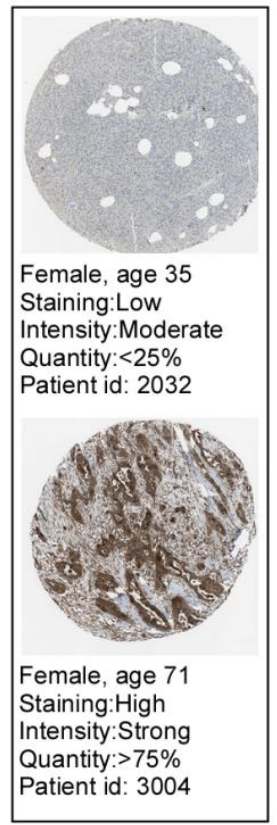

I

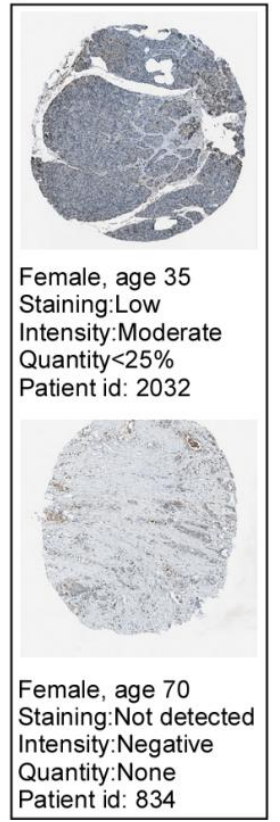

E

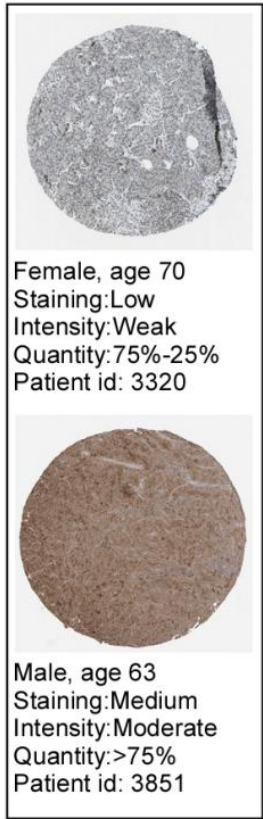

J

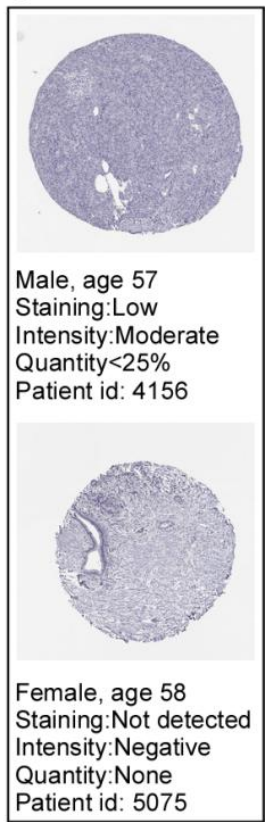


FIGURE 8 Genetic alterations of ten hub genes in the TCGA pancreatic cancer study using the cBioPortal database. a Genetic alteration frequency of hub genes. Different colors represent different kinds of genetic alterations. b A visual summary of alterations of hub genes in per sample. Each sample is presented in a column with each gene in a row. Different kinds of genetic alterations are highlighted in different colors

A

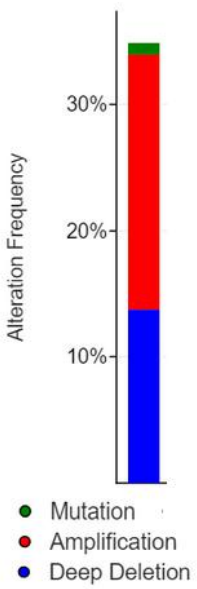

B

EZH2
CDC20
CCNA2
KIF11
UBE2C
DLG3
GPT2
PLCB1
CXCL12
GNG7

Genetic Alteration

study of origin
| 1.2\% .

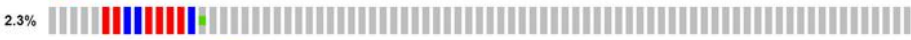

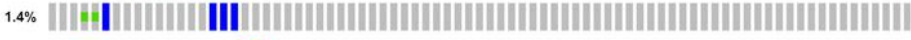
๑.9\% |ПНН

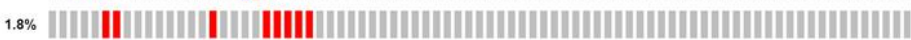

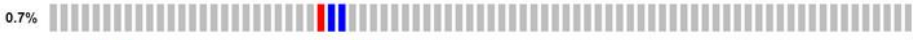

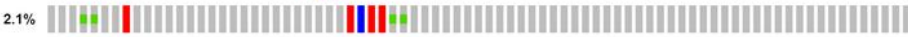

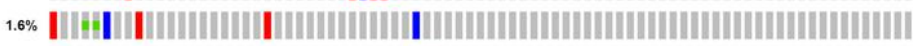

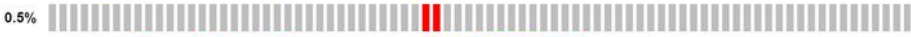

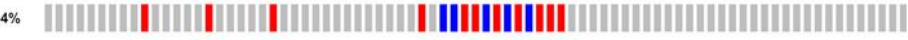

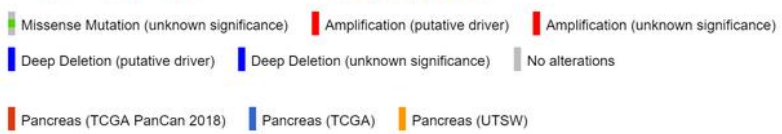


FIGURE 9 The survival analysis of hub gene in pancreatic cancer. (TCGA database)

The mRNA expression levels of (b) CDC20, (d) KIF11, (e) UBE2C have a significant negative correlation with the overall survival of the patients. And the mRNA expression levels of $(\mathrm{h})$

PLCB1, (j) GNG7 have a significant positive correlation with the overall survival according to the GEPIA databases.
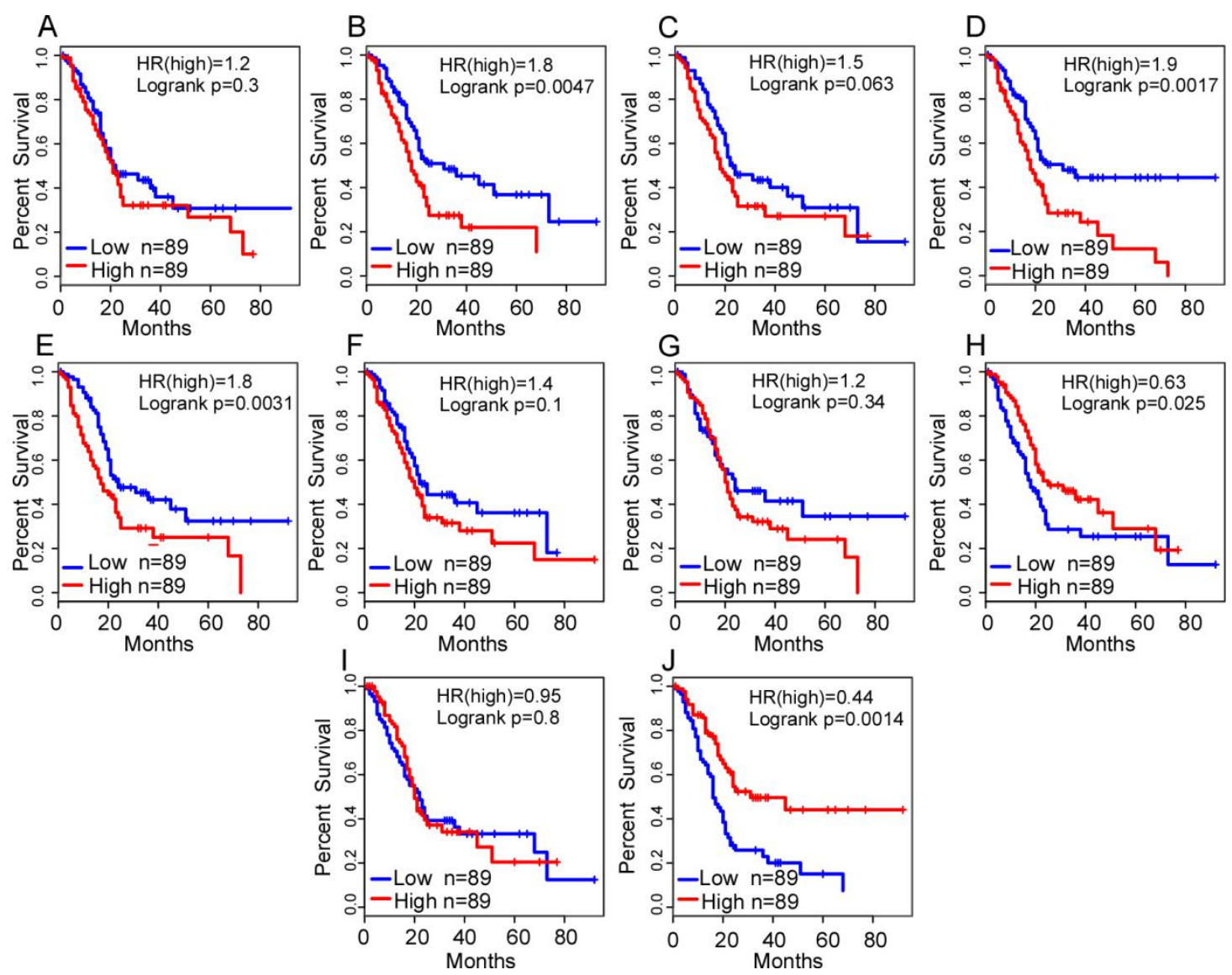
Table. 1 Gene ontology analysis of aberrantly methylated-differentially expressed genes in pancreatic cancer

Abbreviation: Hypo-HGs: high-expression-hypomethylation gene,

Hyper-LGs: low-expression-hypermethylation genes

\begin{tabular}{|c|c|c|c|c|}
\hline \multirow{16}{*}{$\begin{array}{l}\text { Hypo- } \\
\text { HGs }\end{array}$} & Category & Term & Count & PValue \\
\hline & GOTERM_BP_FAT & GO:0007155 cell adhesion & 64 & $2.66 \mathrm{E}-12$ \\
\hline & GOTERM_BP_FAT & GO:0022610 biological adhesion & 64 & $3.11 \mathrm{E}-12$ \\
\hline & GOTERM_BP_FAT & $\begin{array}{l}\text { GO:0009966 regulation of signal } \\
\text { transduction }\end{array}$ & 82 & $8.57 \mathrm{E}-11$ \\
\hline & GOTERM_BP_FAT & $\begin{array}{l}\text { GO:0000904 cell morphogenesis involved } \\
\text { in differentiation }\end{array}$ & 38 & $1.03 \mathrm{E}-10$ \\
\hline & GOTERM_BP_FAT & GO:0023051 regulation of signaling & 88 & $1.40 \mathrm{E}-10$ \\
\hline & GOTERM_CC_FAT & GO:0030055 cell-substrate junction & 22 & $1.51616 \mathrm{E}-06$ \\
\hline & GOTERM_CC_FAT & GO:0005925 focal adhesion & 21 & 4.17596E-06 \\
\hline & GOTERM_CC_FAT & $\begin{array}{l}\text { GO:0005924 cell-substrate adherens } \\
\text { junction }\end{array}$ & 21 & 4.70585E-06 \\
\hline & GOTERM_CC_FAT & GO:0005912 adherens junction & 29 & $5.12621 \mathrm{E}-06$ \\
\hline & GOTERM_CC_FAT & GO:0015629 actin cytoskeleton & 23 & $5.15228 \mathrm{E}-06$ \\
\hline & GOTERM_MF_FAT & GO:0016301 kinase activity & 31 & $6.80019 \mathrm{E}-05$ \\
\hline & GOTERM_MF_FAT & $\begin{array}{l}\text { GO:0050839 cell adhesion molecule } \\
\text { binding }\end{array}$ & 20 & $8.09768 \mathrm{E}-05$ \\
\hline & GOTERM_MF_FAT & GO:0032403 protein complex binding & 26 & 0.000265566 \\
\hline & GOTERM_MF_FAT & $\begin{array}{l}\text { GO:0097367 carbohydrate derivative } \\
\text { binding }\end{array}$ & 55 & 0.000302624 \\
\hline & GOTERM_MF_FAT & $\begin{array}{l}\text { GO:0044877 macromolecular complex } \\
\text { binding }\end{array}$ & 37 & 0.000334021 \\
\hline \multirow{12}{*}{$\begin{array}{l}\text { Hyper- } \\
\text { LGs }\end{array}$} & GOTERM_BP_FAT & GO:0006811 ion transport & 38 & $1.13296 \mathrm{E}-06$ \\
\hline & GOTERM_BP_FAT & GO:0055085 transmembrane transport & 34 & $6.83056 \mathrm{E}-06$ \\
\hline & GOTERM_BP_FAT & GO:0003007 heart morphogenesis & 12 & 4.88161E-05 \\
\hline & GOTERM_BP_FAT & $\begin{array}{l}\text { GO:0019752 carboxylic acid metabolic } \\
\text { process }\end{array}$ & 23 & 0.000110397 \\
\hline & GOTERM_BP_FAT & GO:0043436 oxoacid metabolic process & 23 & 0.000120402 \\
\hline & GOTERM_CC_FAT & GO:0030133 transport vesicle & 13 & $5.96 \mathrm{E}-04$ \\
\hline & GOTERM_CC_FAT & GO:0045202 synapse & 20 & $9.45 \mathrm{E}-04$ \\
\hline & GOTERM_CC_FAT & GO:0097458 neuron part & 28 & 0.002187791 \\
\hline & GOTERM_CC_FAT & $\begin{array}{l}\text { GO:0031226 intrinsic component of } \\
\text { plasma membrane }\end{array}$ & 31 & 0.009300932 \\
\hline & GOTERM_CC_FAT & GO:0005923 bicellular tight junction & 6 & 0.009336207 \\
\hline & GOTERM_MF_FAT & $\begin{array}{l}\text { GO:0019199 transmembrane receptor } \\
\text { protein kinase activity }\end{array}$ & 7 & $3.10 \mathrm{E}-04$ \\
\hline & GOTERM_MF_FAT & GO:0004714 transmembrane receptor & 6 & $7.91 \mathrm{E}-04$ \\
\hline
\end{tabular}




\begin{tabular}{|l|l|l|l|}
\hline & protein tyrosine kinase activity & & \\
\hline GOTERM_MF_FAT & $\begin{array}{l}\text { GO:0005007 fibroblast growth } \\
\text { factor-activated receptor activity }\end{array}$ & 3 & 0.001214922 \\
\hline GOTERM_MF_FAT & GO:0005516 calmodulin binding & 9 & 0.001299847 \\
\hline GOTERM_MF_FAT & $\begin{array}{l}\text { GO:0015171 amino acid transmembrane } \\
\text { transporter activity }\end{array}$ & 6 & 0.0019044 \\
\hline
\end{tabular}

Table. 2 KEGG pathway analysis of aberrantly methylated-differentially expressed genes in pancreatic cancer

\begin{tabular}{|c|c|c|c|c|}
\hline \multirow{6}{*}{$\begin{array}{l}\text { Нypo-H } \\
\text { Gs }\end{array}$} & Category & Term & Count & $P$ Value \\
\hline & $\begin{array}{l}\text { KEGG_PAT } \\
\text { HWAY }\end{array}$ & hsa04512: ECM-receptor interaction & 11 & 2.69E-06 \\
\hline & $\begin{array}{l}\text { KEGG_PAT } \\
\text { HWAY }\end{array}$ & hsa04510: Focal adhesion & 14 & $6.60 \mathrm{E}-05$ \\
\hline & $\begin{array}{l}\text { KEGG_PAT } \\
\text { HWAY }\end{array}$ & hsa05166: HTLV-I infection & 14 & $5.28 \mathrm{E}-04$ \\
\hline & $\begin{array}{l}\text { KEGG_PAT } \\
\text { HWAY }\end{array}$ & hsa04145: Phagosome & 10 & 0.001314404 \\
\hline & $\begin{array}{l}\text { KEGG_PAT } \\
\text { HWAY }\end{array}$ & hsa04151: PI3K-Akt signaling pathway & 15 & 0.003027575 \\
\hline \multirow{5}{*}{$\begin{array}{l}\text { Hyper- } \\
\text { LGs }\end{array}$} & $\begin{array}{l}\text { KEGG_PAT } \\
\text { HWAY }\end{array}$ & hsa00260: Glycine, serine and threonine metabolism & 7 & 7.01169E-06 \\
\hline & $\begin{array}{l}\text { KEGG_PAT } \\
\text { HWAY }\end{array}$ & hsa04972: Pancreatic secretion & 8 & 0.00013766 \\
\hline & $\begin{array}{l}\text { KEGG_PAT } \\
\text { HWAY }\end{array}$ & hsa01230: Biosynthesis of amino acids & 6 & 0.001870579 \\
\hline & $\begin{array}{l}\text { KEGG_PAT } \\
\text { HWAY }\end{array}$ & hsa04964: Proximal tubule bicarbonate reclamation & 4 & 0.002697363 \\
\hline & $\begin{array}{l}\text { KEGG_PAT } \\
\text { HWAY }\end{array}$ & hsa04950: Maturity onset diabetes of the young & 4 & 0.00385578 \\
\hline
\end{tabular}

Abbreviation: Hypo-HGs: high-expression-hypomethylation gene, Hyper-LGs: low-expression-hypermethylation genes 
Table. 3 Module analysis of the protein-protein interaction network

\begin{tabular}{|c|c|c|c|c|c|}
\hline & & Category & Term & Count & $P$ Value \\
\hline \multirow{7}{*}{$\begin{array}{l}\text { Hyp } \\
\text { o-H } \\
\text { Gs }\end{array}$} & $\begin{array}{l}\text { Mo } \\
\text { dle1 }\end{array}$ & $\begin{array}{l}\text { KEGG_PAT } \\
\text { HWAY }\end{array}$ & hsa04110: Cell cycle & 4 & $1.10 \mathrm{E}-04$ \\
\hline & \multirow{3}{*}{$\begin{array}{l}\text { Mo } \\
\text { dle2 }\end{array}$} & $\begin{array}{l}\text { KEGG_PAT } \\
\text { HWAY }\end{array}$ & hsa04512: ECM-receptor interaction & 9 & 4.72E-16 \\
\hline & & $\begin{array}{l}\text { KEGG_PAT } \\
\text { HWAY }\end{array}$ & hsa04510: Focal adhesion & 8 & $1.52 \mathrm{E}-10$ \\
\hline & & $\begin{array}{l}\text { KEGG_PAT } \\
\text { HWAY }\end{array}$ & hsa04151: PI3K-Akt signaling pathway & 8 & $5.76 \mathrm{E}-09$ \\
\hline & \multirow{3}{*}{$\begin{array}{l}\text { Mo } \\
\text { dle3 }\end{array}$} & $\begin{array}{l}\text { KEGG_PAT } \\
\text { HWAY }\end{array}$ & hsa05168: Herpes simplex infection & 4 & $1.85 \mathrm{E}-05$ \\
\hline & & $\begin{array}{l}\text { KEGG_PAT } \\
\text { HWAY }\end{array}$ & hsa05203: Viral carcinogenesis & 3 & $\begin{array}{l}0.0025994 \\
77\end{array}$ \\
\hline & & $\begin{array}{l}\text { KEGG_PAT } \\
\text { HWAY }\end{array}$ & hsa04144: Endocytosis & 3 & $\begin{array}{l}0.0035824 \\
61\end{array}$ \\
\hline \multirow{3}{*}{$\begin{array}{l}\text { Hyp } \\
\text { er-L } \\
\text { Gs }\end{array}$} & \multirow{3}{*}{$\begin{array}{l}\text { Mo } \\
\text { dle1 }\end{array}$} & $\begin{array}{l}\text { KEGG_PAT } \\
\text { HWAY }\end{array}$ & hsa04062: Chemokine signaling pathway & 4 & $1.95 \mathrm{E}-05$ \\
\hline & & $\begin{array}{l}\text { KEGG_PAT } \\
\text { HWAY }\end{array}$ & hsa05200: Pathways in cancer & 4 & $1.85 \mathrm{E}-04$ \\
\hline & & $\begin{array}{l}\text { KEGG_PAT } \\
\text { HWAY }\end{array}$ & hsa04713: Circadian entrainment & 3 & $5.61 \mathrm{E}-04$ \\
\hline
\end{tabular}


References

1. Siegel RL, Miller KD, Jemal A: Cancer statistics, 2018. CA: a cancer journal for clinicians 2018, 68(1):7-30.

2. Lee JH, Giovannetti E, Hwang JH, Petrini I, Wang Q, Voortman J, Wang Y, Steinberg SM, Funel N, Meltzer PS et al: Loss of 18q22.3 involving the carboxypeptidase of glutamate-like gene is associated with poor prognosis in resected pancreatic cancer. Clinical cancer research : an official journal of the American Association for Cancer Research 2012, 18(2):524-533.

3. Xie VK, Li Z, Yan Y, Jia Z, Zuo X, Ju Z, Wang J, Du J, Xie D, Xie K et al: DNA-Methyltransferase 1 Induces Dedifferentiation of Pancreatic Cancer Cells through Silencing of Krüppel-Like Factor 4 Expression. Clinical cancer research : an official journal of the American Association for Cancer Research 2017, 23(18):5585-5597.

4. Yamamoto K, Tateishi K, Kudo Y, Sato T, Yamamoto S, Miyabayashi K, Matsusaka K, Asaoka Y, Ijichi H, Hirata Y et al: Loss of histone demethylase KDM6B enhances aggressiveness of pancreatic cancer through downregulation of C/EBP $\boldsymbol{\alpha}$. Carcinogenesis 2014, 35(11):2404-2414.

5. Botla SK, Savant S, Jandaghi P, Bauer AS, Mücke O, Moskalev EA, Neoptolemos JP, Costello E, Greenhalf W, Scarpa A et al: Early Epigenetic Downregulation of microRNA-192 Expression Promotes Pancreatic Cancer Progression. Cancer Res 2016, 76(14):4149-4159.

6. Baylin SB, Jones PA: Epigenetic Determinants of Cancer. Cold Spring Harbor perspectives in biology 2016, 8(9).

7. Wang YP, Zhou W, Wang J, Huang X, Zuo Y, Wang TS, Gao X, Xu YY, Zou SW, Liu YB et al: Arginine Methylation of MDH1 by CARM1 Inhibits Glutamine Metabolism and Suppresses Pancreatic Cancer. Molecular cell 2016, 64(4):673-687.

8. Kang XC, Chen ML, Yang F, Gao BQ, Yang QH, Zheng WW, Hao S: Promoter methylation and expression of SOCS-1 affect clinical outcome and epithelial-mesenchymal transition in colorectal cancer. Biomedicine $\&$ pharmacotherapy $=$ Biomedecine $\&$ pharmacotherapie 2016, 80:23-29.

9. Kottakis F, Nicolay BN, Roumane A, Karnik R, Gu H, Nagle JM, Boukhali M, Hayward MC, Li YY, Chen T et al: LKB1 loss links serine metabolism to DNA methylation and tumorigenesis. Nature 2016, 539(7629):390-395.

10. Kisiel JB, Raimondo M, Taylor WR, Yab TC, Mahoney DW, Sun Z, Middha S, Baheti S, Zou H, Smyrk TC et al: New DNA Methylation Markers for Pancreatic Cancer: Discovery, Tissue Validation, and Pilot Testing in Pancreatic Juice. Clinical cancer research : an official journal of the American Association for Cancer Research 2015, 21(19):4473-4481.

11. Kanehisa M, Goto S: KEGG: kyoto encyclopedia of genes and genomes. Nucleic acids research 2000, 28(1):27-30.

12. Yu G, Wang LG, Han Y, He QY: clusterProfiler: an R package for comparing biological themes among gene clusters. Omics 2012, 16(5):284-287.

13. Tang Z, Li C, Kang B, Gao G, Li C, Zhang Z: GEPIA: a web server for cancer and normal gene expression profiling and interactive analyses. Nucleic acids research 2017, 45(W1):W98-w102.

14. Saluja A, Maitra A: Pancreatitis and Pancreatic Cancer. Gastroenterology 2019, 156(7):1937-1940. 
15. Xiao Q, Zhou D, Rucki AA, Williams J, Zhou J, Mo G, Murphy A, Fujiwara K, Kleponis J, Salman B et al: Cancer-Associated Fibroblasts in Pancreatic Cancer Are Reprogrammed by Tumor-Induced Alterations in Genomic DNA Methylation. Cancer Res 2016, 76(18):5395-5404.

16. Sandoval J, Heyn H, Moran S, Serra-Musach J, Pujana MA, Bibikova M, Esteller M: Validation of a DNA methylation microarray for 450,000 CpG sites in the human genome. Epigenetics 2011, 6(6):692-702.

17. Liu Y, Jin X, Li Y, Ruan Y, Lu Y, Yang M, Lin D, Song P, Guo Y, Zhao S et al: DNA methylation of claudin- 6 promotes breast cancer cell migration and invasion by recruiting MeCP2 and deacetylating H3Ac and H4Ac. J Exp Clin Cancer Res 2016, 35(1): 120 .

18. Dandawate P, Ghosh C, Palaniyandi K, Paul S, Rawal S, Pradhan R, Sayed AAA, Choudhury S, Standing D, Subramaniam D et al: The Histone Demethylase KDM3A, Increased in Human Pancreatic Tumors, Regulates Expression of DCLK1 and Promotes Tumorigenesis in Mice. Gastroenterology 2019, 157(6):1646-1659.e1611.

19. Wang P, Chen L, Zhang J, Chen H, Fan J, Wang K, Luo J, Chen Z, Meng Z, Liu L: Methylation-mediated silencing of the miR-124 genes facilitates pancreatic cancer progression and metastasis by targeting Rac1. Oncogene 2014, 33(4):514-524.

20. Sidaway P: Colorectal cancer: CpG island methylation indicates inferior survival outcomes. Nature reviews Clinical oncology 2016, 13(8):464-465.

21. Quemener C, Baud J, Boyé K, Dubrac A, Billottet C, Soulet F, Darlot F, Dumartin L, Sire M, Grepin R et al: Dual Roles for CXCL4 Chemokines and CXCR3 in Angiogenesis and Invasion of Pancreatic Cancer. Cancer Res 2016, 76(22):6507-6519.

22. Marinoni I, Wiederkeher A, Wiedmer T, Pantasis S, Di Domenico A, Frank R, Vassella E, Schmitt A, Perren A: Hypo-methylation mediates chromosomal instability in pancreatic NET. Endocrine-related cancer 2017, 24(3):137-146.

23. Simmonds P, Loomis E, Curry E: DNA methylation-based chromatin compartments and ChIP-seq profiles reveal transcriptional drivers of prostate carcinogenesis. Genome medicine 2017, 9(1):54.

24. Bassagañas S, Carvalho S, Dias AM, Pérez-Garay M, Ortiz MR, Figueras J, Reis CA, Pinho SS, Peracaula R: Pancreatic cancer cell glycosylation regulates cell adhesion and invasion through the modulation of $\alpha 2 \beta 1$ integrin and E-cadherin function. PloS one 2014, 9(5):e98595.

25. Zheng X, Carstens JL, Kim J, Scheible M, Kaye J, Sugimoto H, Wu CC, LeBleu VS, Kalluri $\mathrm{R}$ : Epithelial-to-mesenchymal transition is dispensable for metastasis but induces chemoresistance in pancreatic cancer. Nature 2015, 527(7579):525-530.

26. Chen NM, Singh G, Koenig A, Liou GY, Storz P, Zhang JS, Regul L, Nagarajan S, Kühnemuth B, Johnsen SA et al: NFATc1 Links EGFR Signaling to Induction of Sox9 Transcription and Acinar-Ductal Transdifferentiation in the Pancreas. Gastroenterology 2015, 148(5):1024-1034.e1029.

27. Korc M: p38 MAPK in pancreatic cancer: finding a protective needle in the haystack. Clinical cancer research : an official journal of the American Association for Cancer Research 2014, 20(23):5866-5868. 
28. Chen Y, Bai X, Zhang Q, Wen L, Su W, Fu Q, Sun X, Lou Y, Yang J, Zhang J et al: The hepatitis $B$ virus $X$ protein promotes pancreatic cancer through modulation of the PI3K/AKT signaling pathway. Cancer letters 2016, 380(1):98-105.

29. Hasegawa S, Nagano H, Konno M, Eguchi H, Tomokuni A, Tomimaru Y, Asaoka T, Wada H, Hama N, Kawamoto $\mathrm{K}$ et al: A crucial epithelial to mesenchymal transition regulator, Sox4/Ezh2 axis is closely related to the clinical outcome in pancreatic cancer patients. International journal of oncology 2016, 48(1):145-152.

30. Chen Y, Xie D, Yin Li W, Man Cheung C, Yao H, Chan CY, Chan CY, Xu FP, Liu YH, Sung JJ et al: RNAi targeting EZH2 inhibits tumor growth and liver metastasis of pancreatic cancer in vivo. Cancer letters 2010, 297(1):109-116.

31. Chang DZ, Ma Y, Ji B, Liu Y, Hwu P, Abbruzzese JL, Logsdon C, Wang H: Increased CDC20 expression is associated with pancreatic ductal adenocarcinoma differentiation and progression. Journal of hematology \& oncology 2012, 5:15.

32. Gopinathan L, Tan SL, Padmakumar VC, Coppola V, Tessarollo L, Kaldis P: Loss of Cdk2 and cyclin A2 impairs cell proliferation and tumorigenesis. Cancer Res 2014, 74(14):3870-3879.

33. Venere M, Horbinski C, Crish JF, Jin X, Vasanji A, Major J, Burrows AC, Chang C, Prokop J, $\mathrm{Wu} \mathrm{Q}$ et al: The mitotic kinesin KIF11 is a driver of invasion, proliferation, and self-renewal in glioblastoma. Science translational medicine 2015, 7(304):304ra143.

34. Schneider MA, Christopoulos P, Muley T, Warth A, Klingmueller U, Thomas M, Herth FJ, Dienemann H, Mueller NS, Theis F et al: AURKA, DLGAP5, TPX2, KIF11 and CKAP5: Five specific mitosis-associated genes correlate with poor prognosis for non-small cell lung cancer patients. International journal of oncology 2017, 50(2):365-372.

35. Daigo K, Takano A, Thang PM, Yoshitake Y, Shinohara M, Tohnai I, Murakami Y, Maegawa J, Daigo Y: Characterization of KIF11 as a novel prognostic biomarker and therapeutic target for oral cancer. International journal of oncology 2018, 52(1):155-165.

36. Rawat A, Gopal G, Selvaluxmy G, Rajkumar T: Inhibition of ubiquitin conjugating enzyme UBE2C reduces proliferation and sensitizes breast cancer cells to radiation, doxorubicin, tamoxifen and letrozole. Cellular oncology (Dordrecht) 2013, 36(6):459-467.

37. Zhang J, Liu X, Yu G, Liu L, Wang J, Chen X, Bian Y, Ji Y, Zhou X, Chen Y et al: UBE2C Is a Potential Biomarker of Intestinal-Type Gastric Cancer With Chromosomal Instability. Front Pharmacol 2018, 9:847.

38. Saloman JL, Albers KM, Li D, Hartman DJ, Crawford HC, Muha EA, Rhim AD, Davis BM: Ablation of sensory neurons in a genetic model of pancreatic ductal adenocarcinoma slows initiation and progression of cancer. Proc Natl Acad Sci U S A 2016, 113(11):3078-3083.

39. Son J, Lyssiotis CA, Ying H, Wang X, Hua S, Ligorio M, Perera RM, Ferrone CR, Mullarky E, Shyh-Chang $\mathrm{N}$ et al: Glutamine supports pancreatic cancer growth through a KRAS-regulated metabolic pathway. Nature 2013, 496(7443):101-105.

40. Kojima T, Takasawa A, Kyuno D, Ito T, Yamaguchi H, Hirata K, Tsujiwaki M, Murata M, Tanaka S, Sawada N: Downregulation of tight junction-associated MARVEL protein marvelD3 during epithelial-mesenchymal transition in human pancreatic cancer cells. Experimental cell research 2011, 317(16):2288-2298. 
41. Liu Z, Niu Y, Xie M, Bu Y, Yao Z, Gao C: Gene expression profiling analysis reveals that DLG3 is down-regulated in glioblastoma. Journal of neuro-oncology 2014, 116(3):465-476.

42. Roy I, Zimmerman NP, Mackinnon AC, Tsai S, Evans DB, Dwinell MB: CXCL12 chemokine expression suppresses human pancreatic cancer growth and metastasis. PloS one 2014, 9(3):e90400.

43. Guo Y, Scarlata $\mathrm{S}$ : A loss in cellular protein partners promotes $\boldsymbol{\alpha}$-synuclein aggregation in cells resulting from oxidative stress. Biochemistry 2013, 52(22):3913-3920.

44. Cao Y, Lin SH, Wang Y, Chin YE, Kang L, Mi J: Glutamic Pyruvate Transaminase GPT2 Promotes Tumorigenesis of Breast Cancer Cells by Activating Sonic Hedgehog Signaling. Theranostics 2017, 7(12):3021-3033.

45. Manu KA, Chai TF, Teh JT, Zhu WL, Casey PJ, Wang M: Inhibition of Isoprenylcysteine Carboxylmethyltransferase Induces Cell-Cycle Arrest and Apoptosis through p21 and p21-Regulated BNIP3 Induction in Pancreatic Cancer. Molecular cancer therapeutics 2017, 16(5):914-923.

46. Yilmaz M, Christofori G, Lehembre F: Distinct mechanisms of tumor invasion and metastasis. Trends in molecular medicine 2007, 13(12):535-541.

47. Yang J, Gong X, Ouyang L, He W, Xiao R, Tan L: PREX2 promotes the proliferation, invasion and migration of pancreatic cancer cells by modulating the PI3K signaling pathway. Oncology letters 2016, 12(2):1139-1143. 


\section{Figures}
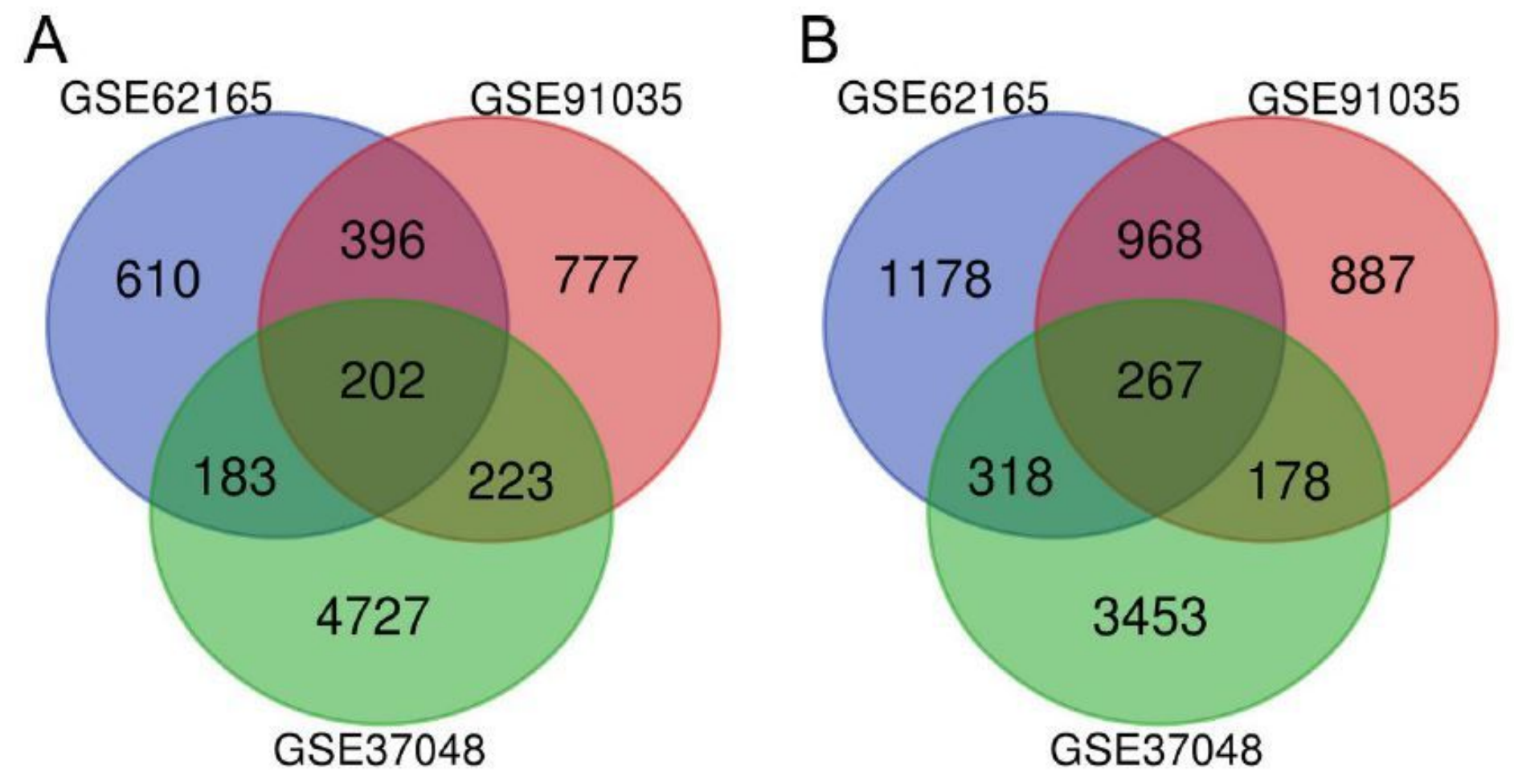

Figure 1

Identification of aberrantly methylated-differentially expressed genes in gene expression datasets (GSE62165, GSE91035) and gene methylation dataset (GSE37048). a. hypomethylation and up-regulated genes; b. hypermethylation and down-regulated genes 


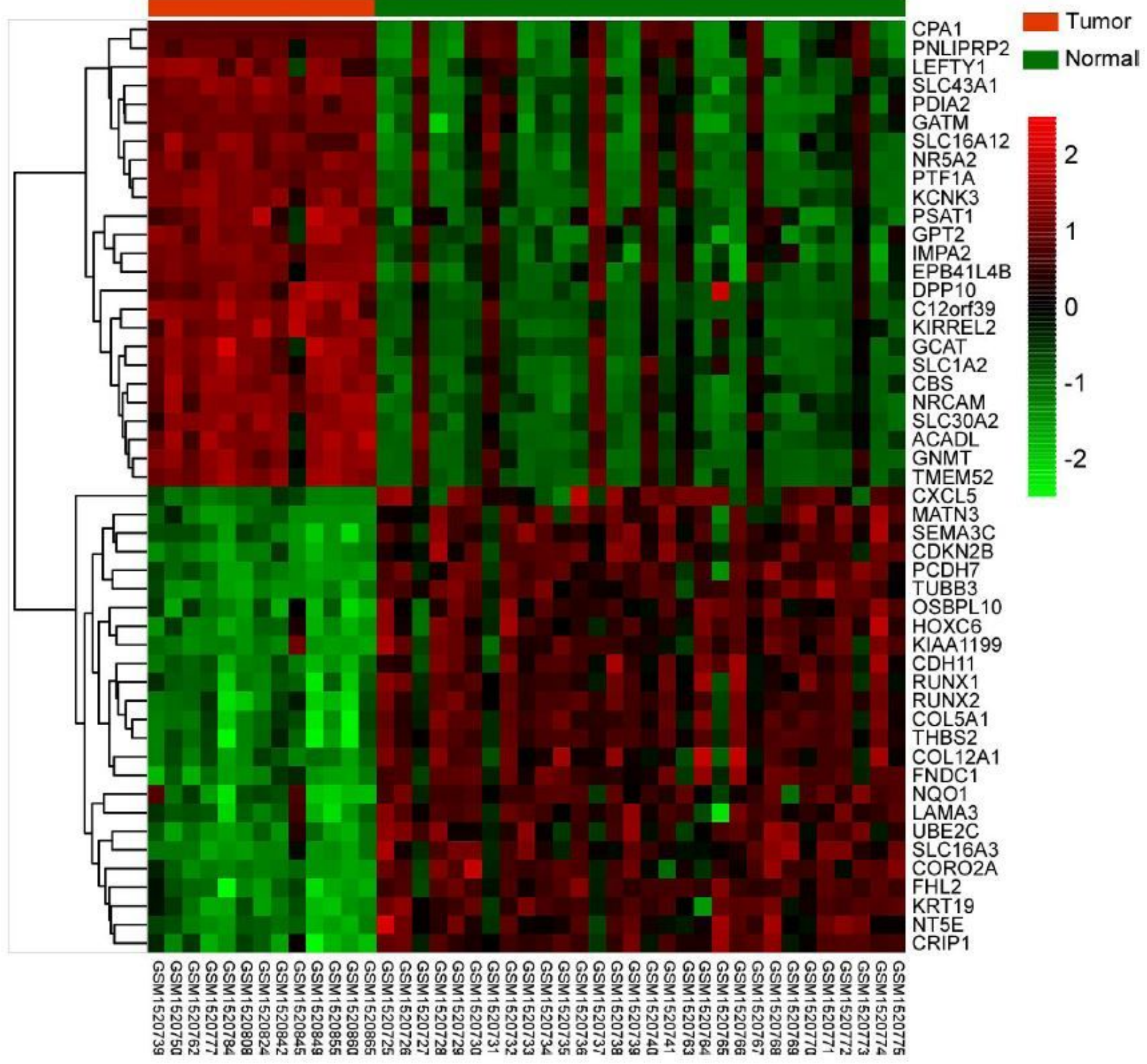

Figure 2

Representative heat map of the top 100 differentially expressed genes in dataset GSE37048 (50 upregulated genes and 50 down-regulated genes). X-axis represents samples, Y-axis represents genes, red stands for upregulation, and green stands for downregulation 


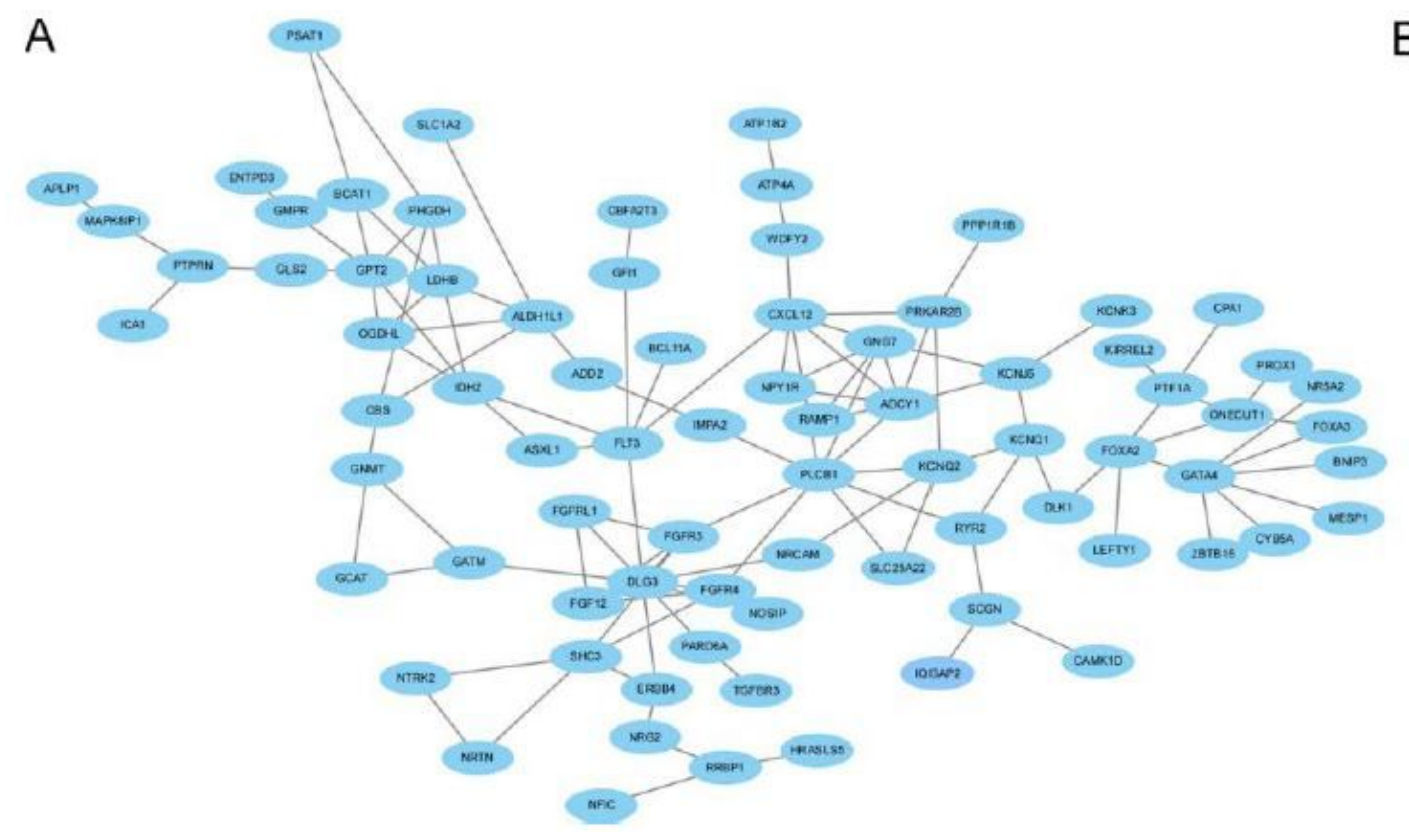

$\mathrm{B}$

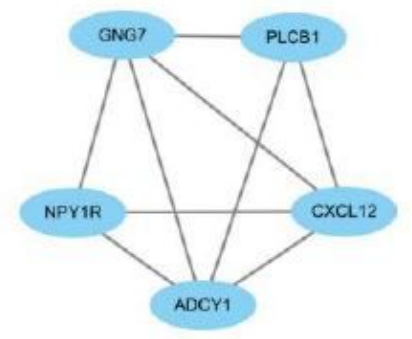

Figure 3

PPI network and modules of hypermethylation-low expression genes. a. PPI network; $b$. top module.

A

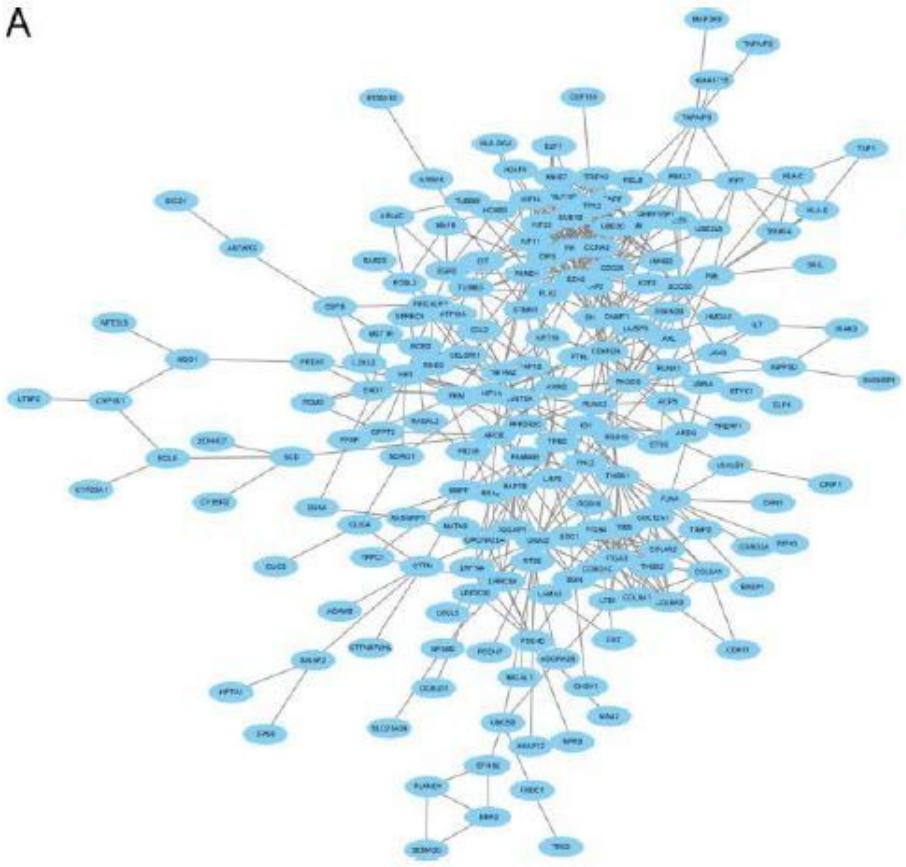

B

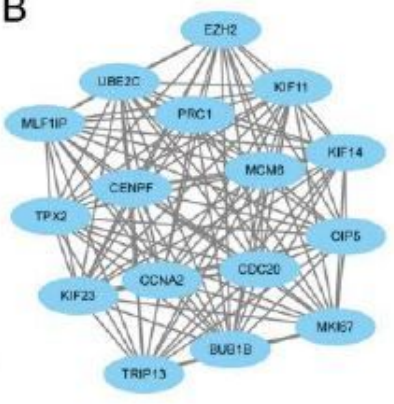

$\mathrm{C}$

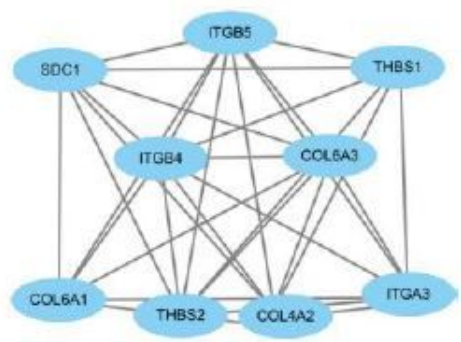

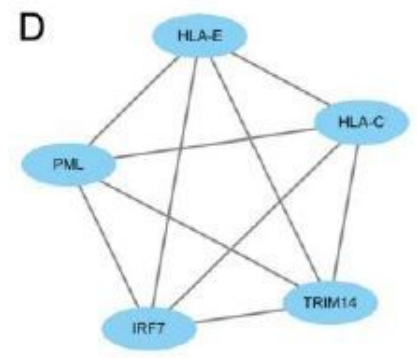

\section{Figure 4}

PPI network and three modules of hypomethylation-high expression on genes. a. PPI network; b.c.d. top module 

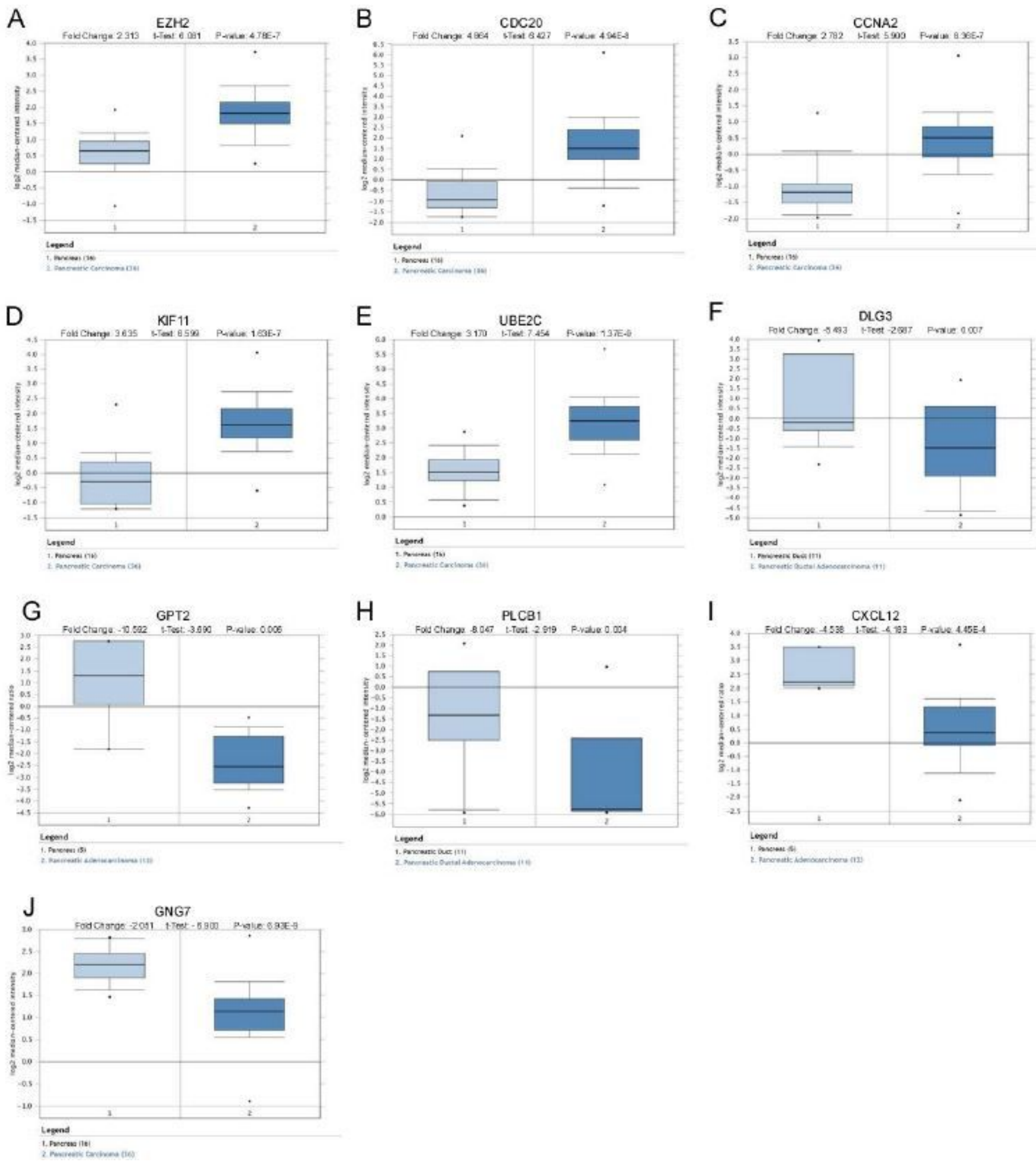

\section{Figure 5}

Validation of the expression of hub genes in Oncomine database. The mRNA expression levels of (a) EZH2, (b) CDC20, (c) CCNA2, (d) KIF11, (e) UBE2C were upregulated in the tumor compared to the normal tissues. And the mRNA expression levels of (f) DLG3, (g) GPT2, (h) PLCB1, (i) CXCL12, (j) GNG7 were down-regulated in the tumor tissues compared to the normal tissues according to the Oncomine databases. 
A

$=$

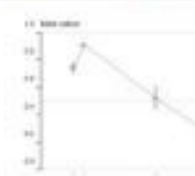

5
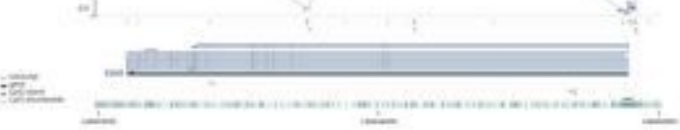

B

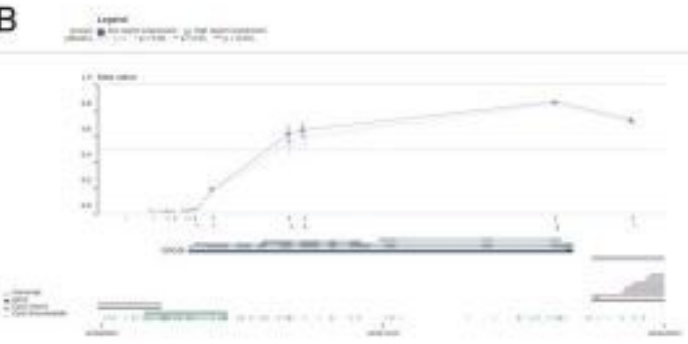

$C=$

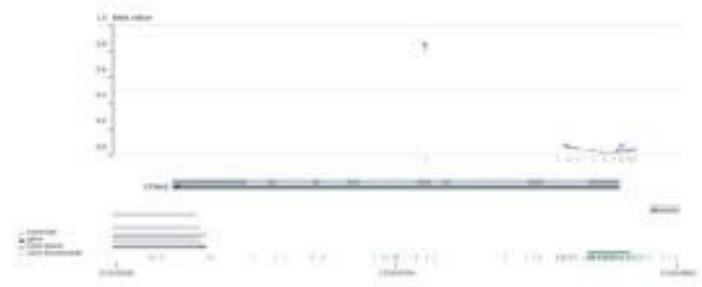

D $=:+\infty=$

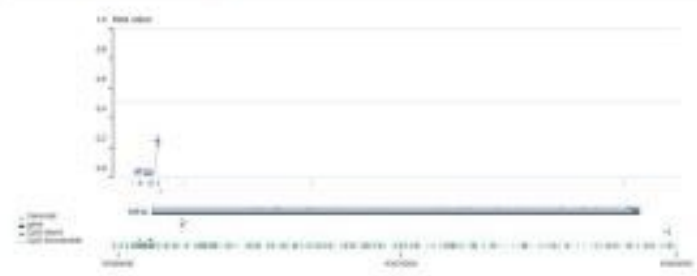

E

$E=:-$

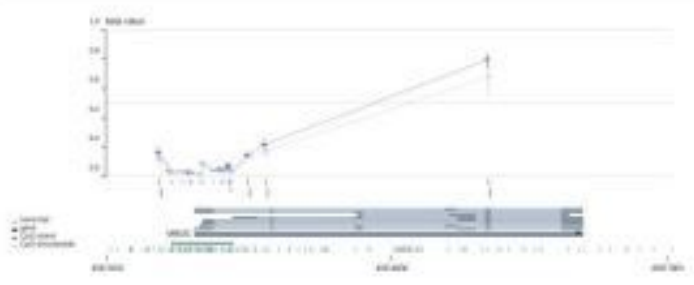

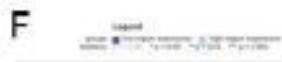
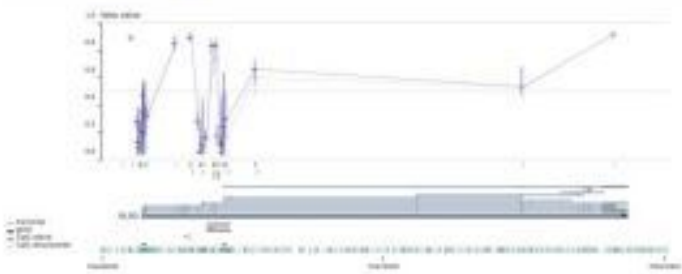

$\mathrm{G}=$

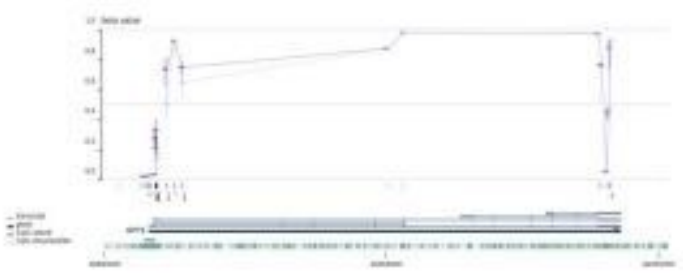

$\mathrm{H}=\mathrm{-}-\mathrm{m}$

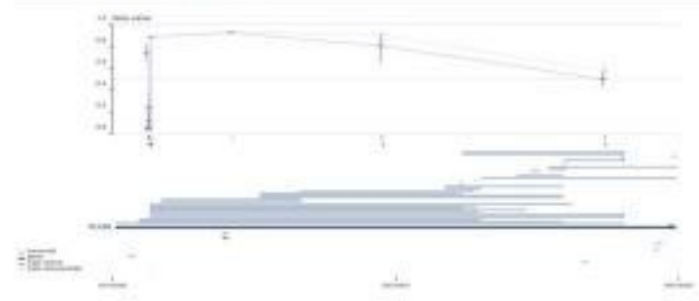

$1 \quad=$
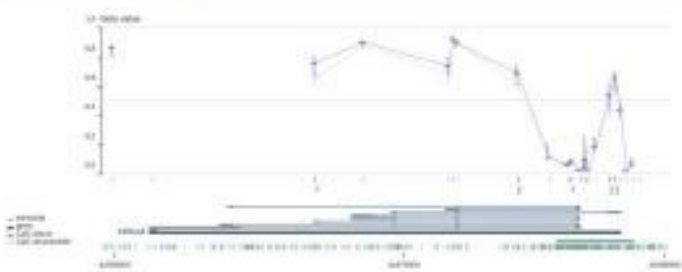

$\mathrm{J}=$

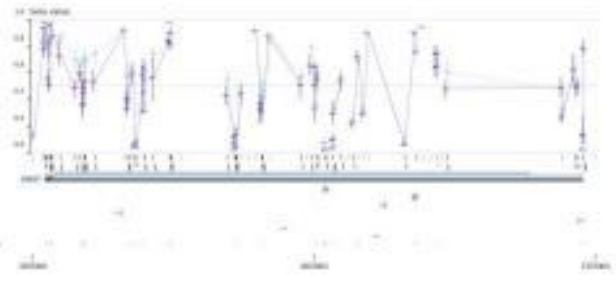

\section{Figure 6}

Verification of the methylation levels of 5 hypomethylated upregulated genes and 5 hypermethylated downregulated genes in MEXPRESS databases. a EZH2. b CDC20. c CCNA2. d KIF11. e UBE2C. f DLG3. g GPT2. h PLCB1. i CXCL12. j GNG7 
A
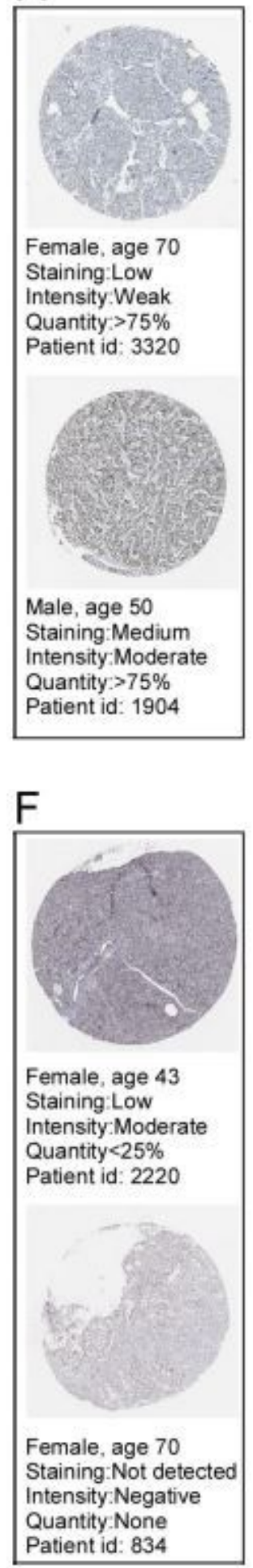

B

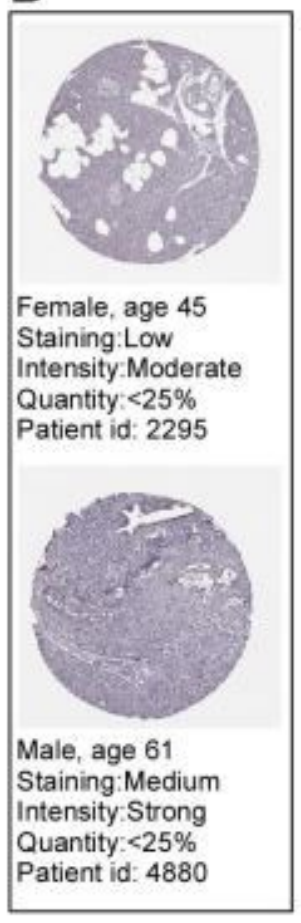

G

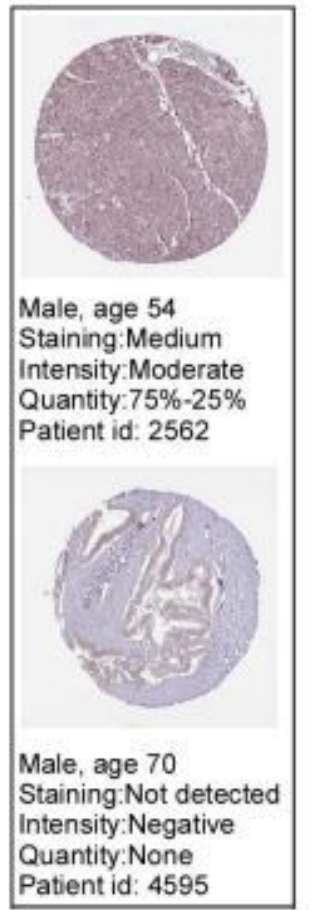

C

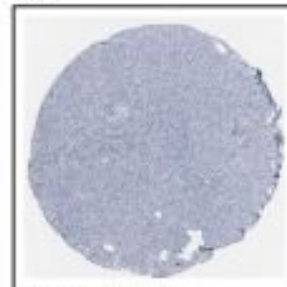

Male, age 66

Staining: Not detected Intensity: Negative

Quantity: None

Patient id: 2329

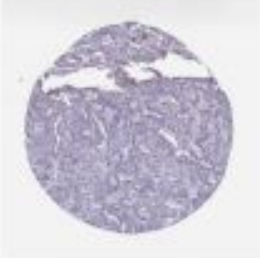

Female, age 61

Staining:Low Intensity:Moderate

Quantity: $<25 \%$

Patient id: 3614
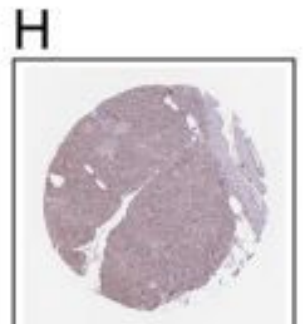

Fermale, age 74

Staining:Weak

Intensity:Moderate

Quantity: $75 \%-25 \%$

Patient id: 2162

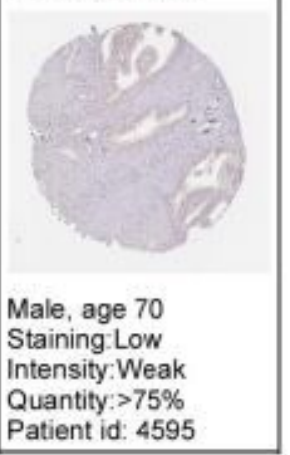

D

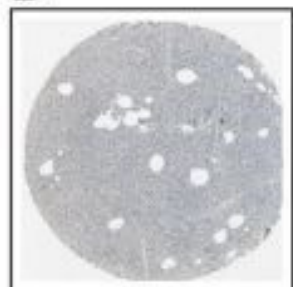

Female, age 35

Staining:Low

Intensity:Moderate

Quantity: $<25 \%$

Patient id: 2032

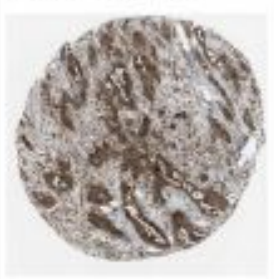

Female, age 71 Staining:High Intensity:Strong Quantity $>75 \%$ Patient id: 3004

।

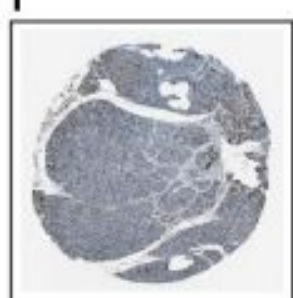

Female, age 35

Staining:Low

Intensity:Moderate

Quantity $<25 \%$

Patient id: 2032

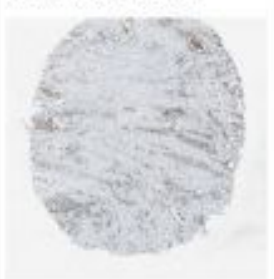

Female, age 70

Staining: Not detected Intensity:Negative

Quantity: None

Patient id: 834
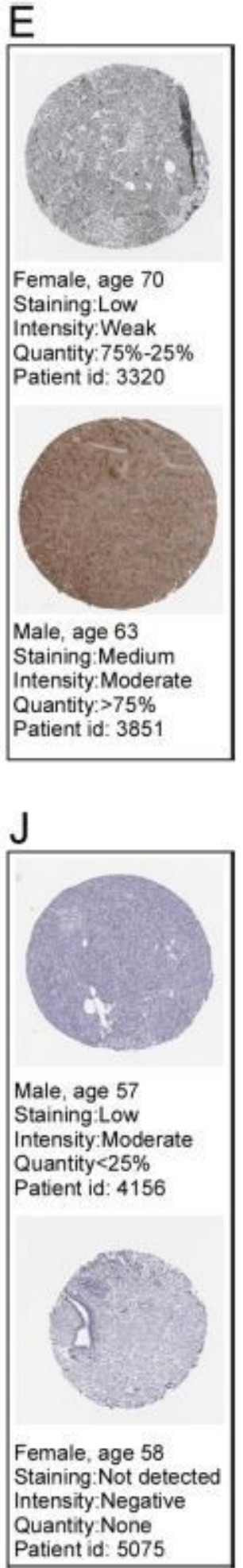

\section{Figure 7}

Validation of hub genes on protein level in the Human Protein Atlas database. The protein levels of (a) EZH2, (b) CDC20, (c) CCNA2, (d) KIF11, (e) UBE2C were upregulated in the tumor compared to the normal tissues. And the protein levels of (f) DLG3, (g) GPT2, (h) PLCB1, (i) CXCL12, (j) GNG7 were down-regulated in the tumor tissues compared to the normal tissues according to the database 


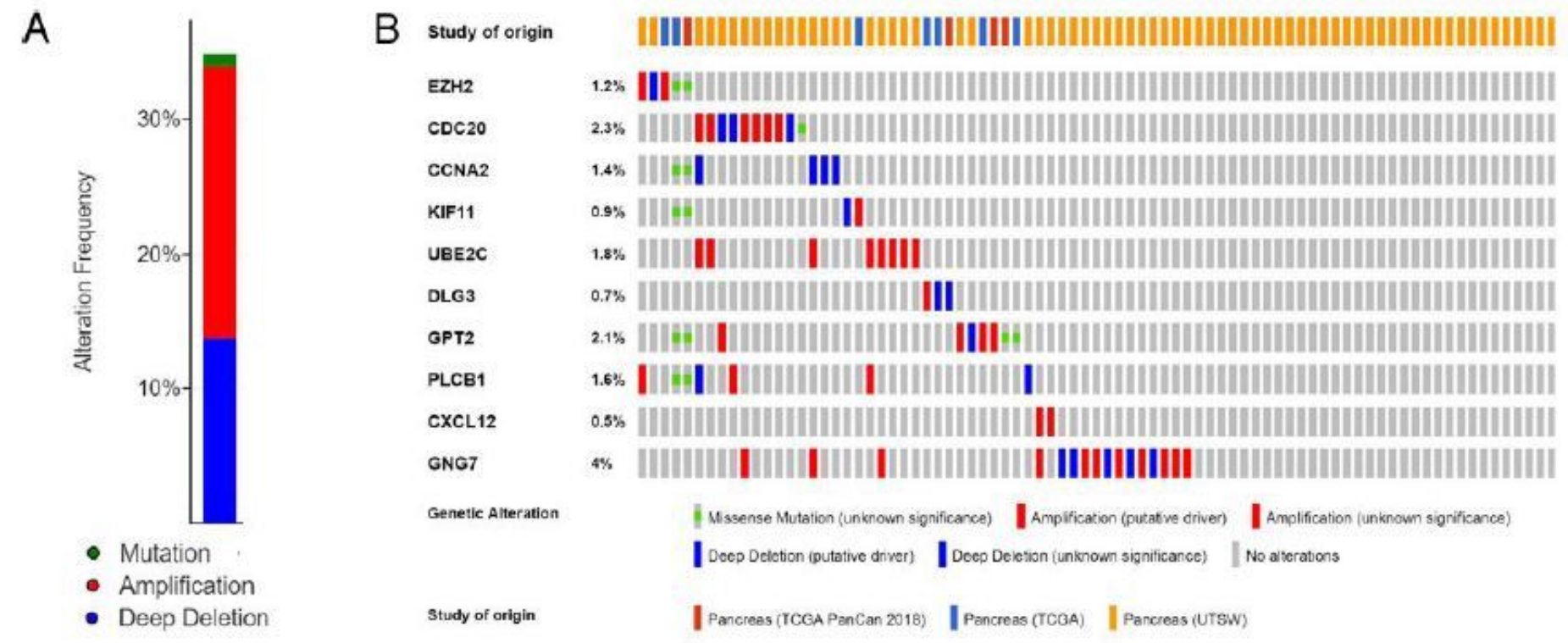

Figure 8

Genetic alterations of ten hub genes in the TCGA pancreatic cancer study using the cBioPortal database. a Genetic alteration frequency of hub genes. Different colors represent different kinds of genetic alterations. b A visual summary of alterations of hub genes in per sample. Each sample is presented in a column with each gene in a row. Different kinds of genetic alterations are highlighted in different colors 

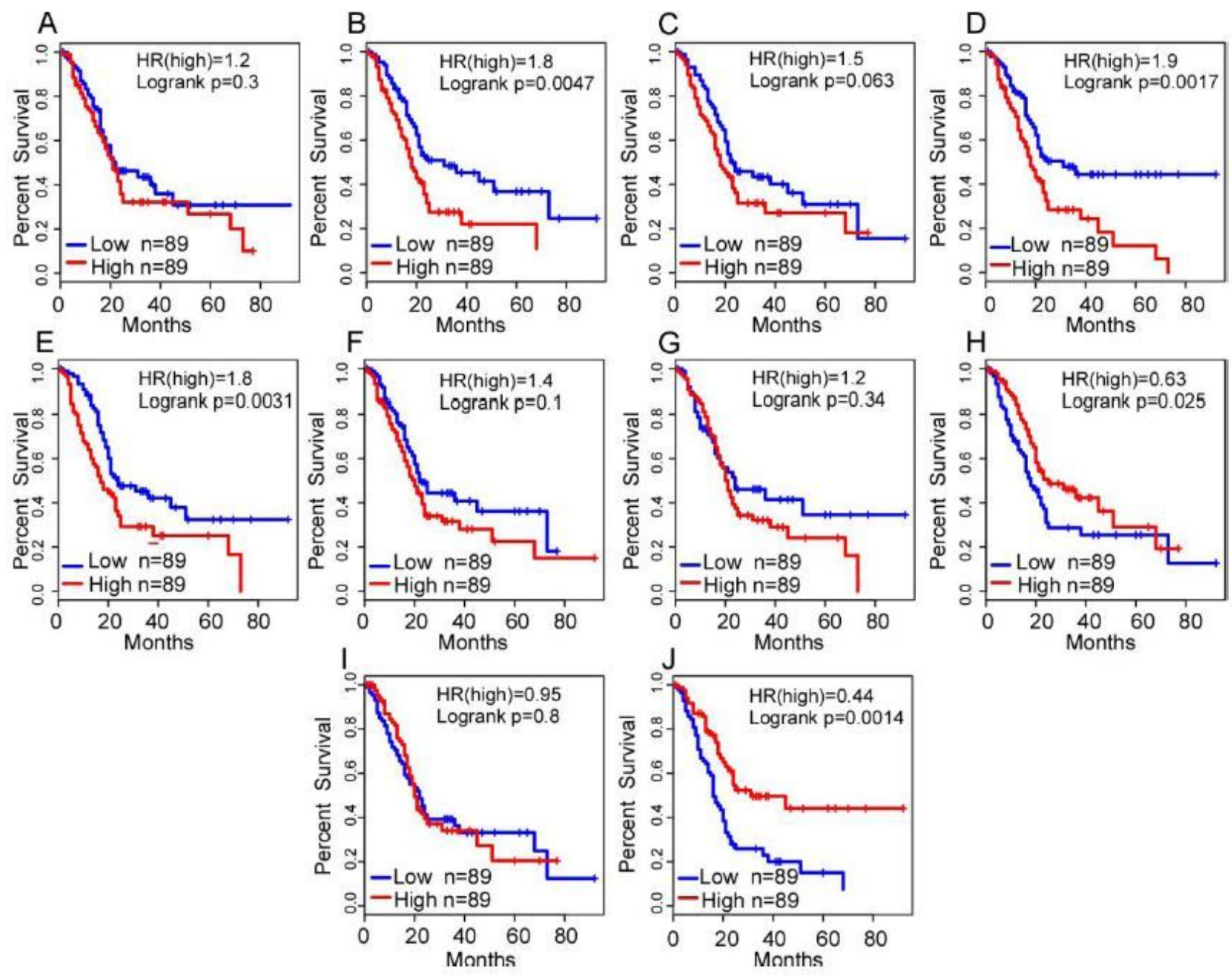

Figure 9

The survival analysis of hub gene in pancreatic cancer. (TCGA database) The mRNA expression levels of (b) CDC20, (d) KIF11, (e) UBE2C have a significant negative correlation with the overall survival of the patients. And the mRNA expression levels of (h) PLCB1, (j) GNG7 have a significant positive correlation with the overall survival according to the GEPIA databases. 